\title{
Finite abelian subgroups of the mapping class group
}

\author{
S ALLEN BRoughton \\ AARON WOOTTON
}

The problem of enumeration of conjugacy classes of finite abelian subgroups of the mapping class group $\mathcal{M}_{\sigma}$ of a closed, smooth, orientable surface $S$ of genus $\sigma \geq 2$ is considered. A complete method of enumeration is achieved for finite elementary abelian subgroups and steps are taken toward enumeration of finite abelian subgroups.

20F34, 20F36, 14H37; 14H30, 14J50

\section{Introduction}

Let $S$ be a closed, smooth, orientable surface of genus $\sigma \geq 2$. The mapping class group $\mathcal{M}_{\sigma}$ of $S$ (or MCG) is the group of isotopy classes of homeomorphisms of $S$. In this paper we shall investigate the conjugacy classes of finite elementary abelian subgroups, and more generally the finite abelian subgroups of $\mathcal{M}_{\sigma}$. While the general finite subgroup classification is important we focus on the case where $G$ is an elementary abelian subgroup as a tractable case where complete classification methods by standard linear algebra is possible. We note that by finite elementary abelian, we mean $G \cong C_{p}^{v} \cong \mathbb{F}_{p}^{v}$ where $C_{p}$ is a cyclic group of prime order $p$, and $\mathbb{F}_{p}$ is the finite field of the same cardinality (we use the latter notation when we want to emphasize the vector space structure of $G$ ). We also consider the abelian case where positive steps can be made toward such a classification. The main result of our work is to describe methods which may be employed to completely classify all elementary abelian actions in a given genus and steps toward a classification of abelian actions. The classification is complex since it involves understanding the representation theory of certain subgroups of symmetric groups, but for a fixed low genus since the symmetric groups are small, we are able to produce very explicit results.

Our main results are presented in three parts. The first part, Theorem 2.8 in Section 2, gives a decomposition of an abelian subgroup $G$ of $\mathcal{M}_{\sigma}$ into an unramified part (hyperbolic) and a ramified part (elliptic). We note that our use of the term "elliptic" is with reference to the type of generating vector of the subgroup (see Section 2.4 for details), in contrast to Bers [2] and Gilman [18] where it is used to refer to finite subgroups of $\mathcal{M}_{\sigma}$. Since all the subgroups we are considering are finite, this should not 
cause any confusion. The second part, Section 3, gives a complete classification of the unramified part for elementary abelian subgroups and provides steps to classify general unramified abelian actions. Though complete results are not obtained for the general abelian case, enough information is gathered to classify unramified abelian actions on surfaces up to genus 65 (see Example 3.8) and the general method of how to classify abelian actions is described through other examples. In the third part, Section 4 , we present methods allowing one to classify the ramified part for an elementary abelian subgroup. We finish in Section 5 with some explicit examples to show how these methods are utilized.

A starting point for our work are the papers Harvey [20] and MacLachlan [27] which discuss cyclic and abelian groups of surface automorphisms and Gilman [16] where the rank 1 elementary abelian subgroups of $\mathcal{M}_{\sigma}$ are considered. In [16], the concept of a generating vector is introduced and this is a concept which we shall make heavy use of in our classification. Our general approach is to adapt and extend the methods of Harvey [21], especially in the discussion of unramified actions, using the correspondence between groups of conformal automorphisms and conjugacy classes of subgroups of the MCG.

We should note that our methods fail for nonabelian groups. There is a general classification theory which describes the classes of subgroups as a finite sequence of quotients of finite sets by the action of other mapping class groups (see Broughton [8] for instance). In the abelian case the general method simplifies greatly because all commutators are trivial, and we get a nice splitting into ramified and unramified cases and the subcases are tractable. For the general case however, these simplifications do not occur and calculations become complicated very quickly and are only really possible through computer calculation. For low genus, some partial results including many cases of infinite families are known, as mentioned further in this introduction.

Our study of finite subgroups of $\mathcal{M}_{\sigma}$ is primarily motivated by the over one hundred year old study of groups of automorphisms of compact Riemann surfaces. Every group of automorphisms of a surface genus $\sigma \geq 2$ has an isomorphic image in $\mathcal{M}_{\sigma}$ by sending an automorphism to its homotopy class. Due to the resolution of the Nielsen Realization Problem by Kerckhoff [23], every finite subgroup of $\mathcal{M}_{\sigma}$ arises this way. Thus the study of automorphisms of Riemann surfaces up to topological equivalence is the same as the study of conjugacy classes of finite subgroups of the mapping class group; see Proposition 2.1 in Section 2 for a precise statement. Early methods for finding automorphism groups were ad hoc and focused on the geometry of the surfaces themselves, with special attention for groups meeting the maximal order bound 84( $\sigma-1)$, the Hurwitz bound. Later in the 1960's and 1970's, a more systematic study began, especially using uniformizing Fuchsian groups. For instance, see the papers of 
Macbeath [25; 26], MacLachlan [27; 28], Harvey [20; 21], Gilman [16; 17], Gilman and Patterson [19], and Singerman [30; 31; 32; 33]. The existence of automorphisms of $S$ guarantees that any uniformizing group $\Pi$ for $S$ lies in some larger Fuchsian group $\Gamma$ as a normal subgroup, and $\Gamma / \Pi \cong G$ (see Section 2 for details). Moreover, $\Gamma$ is a finitely presented group whose presentation is completely determined by data derived from the fixed points of the action of $G$ on $S$. Thus the problem of determination of conjugacy classes of $\mathcal{M}_{\sigma}$ can be interpreted as a problem in computational group theory. For details on this systematic approach, see Section 2.2.

Since the development of this more systematic approach and in part due to the advances in computer algebra systems, there has been tremendous progress in classification results of automorphism groups of compact Riemann surfaces. A review of some results as well as references are given in the paper Broughton [8] and the monograph Breuer [6]. In [8] the complete classification in genus 2 and 3 is given (with one omission; see Broughton [10]). Some additional calculations are available in preprint form; see Broughton, Dirks, Sloughter and Vinroot [11] and Gregoire and Averill [1] for higher genus. In addition, there is much literature dedicated to infinite families of surfaces whose automorphism groups share certain properties; see for example Harvey [20], Wootton [34; 35], Broughton [8], Conder and Kulkarni [13], and Kulkarni [24]. For reference, a comprehensive study in this area is given in Breuer's monograph [6]. In this work he outlines a complete classification up to genus 48 .

We note that in Breuer's monograph [6], group actions are considered equivalent if their representations on the first homology group are equivalent, a coarser classification than the classification up to conjugacy in the mapping class group. These two different ways to classify group actions for cyclic prime groups were considered in Gilman [17] and Gilman and Patterson [19] and more recently in preprint form in Gilman [15]. An interesting related topic would be to compare these two different classifications for other types of groups. Another related topic is the study of the Torelli group $I_{\sigma}$ which is the subgroup of $\mathcal{M}_{\sigma}$ consisting of all isotopy classes which act trivially on the first homology group, and the Torelli Modular group which is defined to be $\mathcal{M}_{\sigma} / I_{\sigma}$. The Torelli Modular group may be identified with $\operatorname{Sp}(2 \sigma, \mathbb{Z})$ under the homology representation $\mathcal{M}_{\sigma} \rightarrow \operatorname{Sp}(2 \sigma, \mathbb{Z})$. Since the Torelli group is torsion-free, the order of an element of finite order in $\mathcal{M}_{\sigma}$ will be preserved in the Torelli Modular group, and consequently there is a nice correspondence between finite subgroups of the Torelli Modular group and those of $\mathcal{M}_{\sigma}$. For more information on the Torelli group and Torelli Modular group, see for example Johnson [22] and Brendle [4].

A second motivating reason for studying the finite subgroups is to describe the structure, especially the singularity structure (nonmanifold points), of the moduli space $\mathfrak{M}_{\sigma}$ of surfaces of genus $\sigma$. The moduli space $\mathfrak{M}_{\sigma}$ of surfaces of genus $\sigma$ is the space 
of conformal equivalence classes of surfaces of genus $\sigma$. The moduli space may be obtained as a quotient $\mathfrak{M}_{\sigma}=\mathfrak{T}_{\sigma} / \mathcal{M}_{\sigma}$, of the Teichmüller space $\mathfrak{T}_{\sigma}$ which is homeomorphic to an open ball in $\mathbb{C}^{3 \sigma-3}$. In this context $\mathcal{M}_{\sigma}$ is often called the Teichmüller modular group or the modular group. The singular points of $\mathfrak{M}_{\sigma}$ (away from the hyperelliptic locus in $\sigma=2,3$ ) are caused by the fixed points of finite subgroups of $\mathcal{M}_{\sigma}$, which in turn controls the torsion cohomology of the mapping class group. For more details and applications, see Broughton [7]. The moduli space may also be constructed from the Torelli group. The Torelli space $\mathfrak{T}_{\sigma} / I_{\sigma}$ is a smooth manifold since $I_{\sigma}$ is torsion-free. Hence there is a faithful action of $\mathcal{M}_{\sigma} / I_{\sigma}$ on the manifold $\mathfrak{T}_{\sigma} / I_{\sigma}$ yielding the quotient $\left(\mathfrak{T}_{\sigma} / I_{\sigma}\right) /\left(\mathcal{M}_{\sigma} / I_{\sigma}\right)=\mathfrak{T}_{\sigma} / \mathcal{M}_{\sigma}=\mathfrak{M}_{\sigma}$.

An especially simple example of the interplay between geometric structure and the finite subgroups of $\mathcal{M}_{\sigma}$ was made apparent when Maclachlan showed in [28] that $\mathcal{M}_{\sigma}$ is generated by torsion elements (and consequently that the quotient space moduli space is simply connected). Following this, a number of different people have found sets of torsion generators for $\mathcal{M}_{\sigma}$; see Birman [3] and Brendle and Farb [5]. An understanding of how such elements generate $\mathcal{M}_{\sigma}$ may be possible through an analysis of the conjugacy classes of finite subgroups of $\mathcal{M}_{\sigma}$.

Acknowledgments The first author wishes to thank I M Isaacs for advice shortening the exposition in Section 4. The second author thanks the University of Portland for support through the Butine faculty support program. Both authors would like to thank the referee for suggestions on improving the introduction and better linking the paper with the literature.

\section{Reduction of classification to finite group calculations}

It is standard to study surface groups, Teichmüller space, and the moduli space in terms of Fuchsian groups so we present our problem in that context.

\subsection{Finite group actions and finite subgroups of $\mathcal{M}_{\sigma}$}

Let $G$ be a finite group. The group $G$ is said to act (in an orientation preserving manner) on surface a $S$ of genus $\sigma \geq 2$ if there is an injection

$$
\epsilon: G \hookrightarrow \operatorname{Homeo}^{+}(S)
$$

into the group of orientation preserving homeomorphisms. Two actions $\epsilon_{1}, \epsilon_{2}$ are said to be topologically equivalent it there is a homeomorphism $h$ of $S$ and an automorphism $\omega$ of $G$ such that

$$
\epsilon_{2}(\omega(g))=h \circ \epsilon_{1}(g) \circ h^{-1} \text {. }
$$

Algebraic $\mathcal{E} \mathcal{G}$ eometric Topology, Volume 7 (2007) 
This is equivalent to saying that the images $\epsilon_{1}(G)$ and $\epsilon_{2}(G)$ are conjugate in the group Homeo $^{+}(S)$. The following are well known for $\sigma \geq 2$ :

- The map to the mapping class group $\mathcal{M}_{\sigma}=\mathcal{M}(S)$ is injective

$$
G \stackrel{\epsilon}{\longrightarrow} \text { Homeo }^{+}(S) \stackrel{\iota}{\longrightarrow} \mathcal{M}_{\sigma}
$$

because $G$ acts faithfully on $H_{1}(S)$.

- Given a finite subgroup $H \subset \mathcal{M}_{\sigma}$ there is a finite group $G \subset \mathrm{Homeo}^{+}(S)$ such that $H=\iota(G)$ and at least one conformal structure on $S$ such that $G$ is a group of conformal automorphisms with respect to this conformal structure (Nielsen Realization problem; see Kerckhoff [23]).

Thus we have the following:

Proposition 2.1 The map induced by (1) is a 1-1 correspondence between topological equivalence classes of $G$-actions and conjugacy classes of subgroups of the mapping class group.

\subsection{Covering Fuchsian group and generating vectors}

Suppose that $G$ acts conformally on $S$. The quotient $T=S / G$ is a surface of some genus $\rho$ and the quotient map $S \rightarrow S / G$ is branched over $r$ points $Q_{1}, \ldots, Q_{r}$ with periods (or branching orders) $m_{1}, \ldots, m_{r}$. We say that $\mathcal{S}=\left(\rho ; m_{1}, \ldots, m_{r}\right)$ is the signature or branching data of $G$ acting on $S$ and that $S$ admits a $G-\left(\rho ; m_{1}, \ldots, m_{r}\right)$ action. Since $G$ acts conformally there is a uniformizing Fuchsian group $\Gamma$ for the $G$-action and the signature of $\Gamma$ is $\left(\rho ; m_{1}, \ldots, m_{r}\right)$. More precisely we have a map

$$
\Pi \hookrightarrow \Gamma \stackrel{\eta}{\rightarrow} G
$$

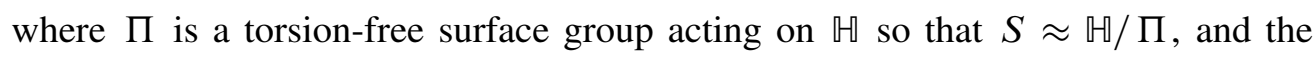
$G$-action on $S$ is induced by the isomorphism $\bar{\eta}: \Gamma / \Pi \longleftrightarrow G$ and the natural action of $\Gamma / \Pi$ on $\mathbb{H} / \Pi$.

Any such epimorphism $\Gamma \stackrel{\eta}{\rightarrow} G$ can be neatly summarized in the context of finite groups by a generating vector. Generating vectors were initially introduced by Gilman [16] for the abelian case to enumerate conjugacy classes of automorphisms of order $p$. See Broughton $[7 ; 8]$ and Breuer [6] for additional details. For the conformal action of $G$ on a surface $S$ with signature $\mathcal{S}=\left(\rho ; m_{1}, \ldots, m_{r}\right)$, the group $\Gamma$ has signature 
$\left(\rho ; m_{1}, \ldots, m_{r}\right)$ and a presentation

$$
\begin{aligned}
& \Gamma \simeq \Gamma\left(\rho ; m_{1}, \ldots, m_{r}\right) \\
& =\left\langle\alpha_{i}, \beta_{i}, \gamma_{j}, 1 \leq i \leq \rho, 1 \leq j \leq r \mid \prod_{i=1}^{\rho}\left[\alpha_{i}, \beta_{i}\right] \prod_{j=1}^{r} \gamma_{j}=\gamma_{1}^{m_{1}}=\cdots=\gamma_{r}^{m_{r}}=1\right\rangle,
\end{aligned}
$$

and the genus $\sigma$ of $S$ is given by the Riemann-Hurwitz equation

$$
\frac{(2 \sigma-2)}{|G|}=(2 \rho-2+r)-\sum_{j=1}^{r} \frac{1}{m_{j}} .
$$

Let $a_{i}, b_{i}, c_{j}$ be images of the generators $\alpha_{i}, \beta_{i}, \gamma_{j}$ under the epimorphism $\eta: \Gamma \longrightarrow G$, ie,

$$
\eta: \alpha_{i} \mapsto a_{i}, \eta: \beta_{i} \mapsto b_{i}, \eta: \gamma_{j} \mapsto c_{j}
$$

Then the set $\left\{a_{i}, b_{i}, c_{j} \mid 1 \leq i \leq \rho, 1 \leq j \leq r\right\}$ is a generating set for $G$ satisfying the following properties:

$$
\begin{gathered}
\prod_{i=1}^{\rho}\left[a_{i}, b_{i}\right] \prod_{j=1}^{r} c_{j}=1, \\
o\left(c_{j}\right)=m_{j} .
\end{gathered}
$$

Each such generating set gives us a $(2 \rho+r)$-tuple $\left(a_{1}, \ldots a_{\rho}, b_{1}, \ldots b_{\rho}, c_{1}, \ldots c_{\rho}\right)$ satisfying (5) and (6) and is called a $\left(\rho ; m_{1}, \ldots, m_{r}\right)$-generating vector. There is a one to one correspondence between the set of $\left(\rho ; m_{1}, \ldots, m_{r}\right)$-generating vectors of $G$ and $\operatorname{Epi}(\Gamma, G)$, epimorphisms $\Gamma \rightarrow G$ preserving the orders of the $\gamma_{j}$, once a generating set $\mathcal{G}=\left\{\alpha_{i}, \beta_{i}, \gamma_{j} \mid 1 \leq i \leq \rho, 1 \leq j \leq r\right\}$ of $\Gamma$ has been fixed. Let $X^{\circ}(G, \mathcal{S})=X^{\circ}\left(G, \rho ; m_{1}, \ldots, m_{r}\right)$ denote the set of $\left(\rho ; m_{1}, \ldots, m_{r}\right)$ - generating vectors of $G$ acting on $S$.

There is a natural action of $\operatorname{Aut}(G) \times \operatorname{Aut}(\Gamma)$ on $\operatorname{Epi}(\Gamma, G)$ by

$$
\eta \longrightarrow \omega \circ \eta \circ \xi^{-1}
$$

where $(\omega, \xi) \in \operatorname{Aut}(G) \times \operatorname{Aut}(\Gamma)$. The action transfers to $X^{\circ}(G, \mathcal{S})$ in a natural way. The following is well known and the justification is provided in some detail in Broughton [8].

Proposition 2.2 Using the notation above, we have:

(1) Each finite subgroup of the mapping class group has a uniquely determined signature which may be recovered from the homology representation. 
(2) The conjugacy classes of finite subgroups of the mapping class group are in one to one correspondence to the $\operatorname{Aut}(G) \times \operatorname{Aut}(\Gamma)$ orbits on $X^{\circ}(G, \mathcal{S})$.

(3) The group Aut $(\Gamma)$ may be constructed geometrically from the mapping class group $\mathcal{M}_{\mathcal{B}}$ of $T$ preserving the branch point set and the branching orders.

Remark 2.3 Let's give a bit more detail on the last point of the proposition. Let $\mathcal{B}=\left\{Q_{1}, \ldots, Q_{r}\right\}$ denote the branch point set, and $T^{o}=T-\mathcal{B}$ and $Q_{0} \in T^{o}$. Every homeomorphism of $T^{o}$ may be identified with a homeomorphism of the pair $(T, \mathcal{B})$ and vice versa. The fundamental group, $\pi_{1}\left(T^{o}, Q_{0}\right)$ has a presentation of the form

$$
\left\langle\widetilde{\alpha}_{i}, \widetilde{\beta}_{i}, \tilde{\gamma}_{j}, 1 \leq i \leq \rho, 1 \leq j \leq r \mid \prod_{i=1}^{\rho}\left[\tilde{\alpha}_{i}, \widetilde{\beta}_{i}\right] \prod_{j=1}^{r} \tilde{\gamma}_{j}=1\right\rangle
$$

where the $\tilde{\alpha}_{i}, \tilde{\beta}_{i}$ are the canonical pair around the $i$-th handle and $\tilde{\gamma}_{j}$ encircles the $j$-th puncture. There is an epimorphism $\pi_{1}\left(T^{o}, Q_{0}\right) \rightarrow \Gamma$ given by

$$
\tilde{\alpha}_{i} \rightarrow \alpha_{i}, \tilde{\beta}_{i} \rightarrow \beta_{i}, \tilde{\gamma}_{j} \rightarrow \gamma_{j}
$$

Let $\mathcal{M}\left(T^{o}, \mathcal{S}\right)$ denote the subgroup of the mapping class group of $\mathcal{M}\left(T^{o}\right)$ that preserves periods of the branch points. Then the canonical map $\mathcal{M}\left(T^{o}, \mathcal{S}\right) \rightarrow \operatorname{Out}(\Gamma)$ is an isomorphism; see Zieschang, Vogt and Coldeway [36] for more details.

\subsection{The $\operatorname{Aut}(G) \times \operatorname{Aut}(\Gamma)$ actions for abelian groups}

It is clear how $\operatorname{Aut}(G)$ acts on $X^{\circ}(G, \mathcal{S})$ namely $\omega \in \operatorname{Aut}(G)$ acts via

$$
\left(a_{1}, \ldots a_{\rho}, b_{1}, \ldots b_{\rho}, c_{1}, \ldots c_{\rho}\right) \rightarrow\left(\omega a_{1}, \ldots \omega a_{\rho}, \omega b_{1}, \ldots \omega b_{\rho}, \omega c_{1}, \ldots \omega c_{r}\right) .
$$

In order to compute the action of $\operatorname{Aut}(\Gamma)$ on $X^{\circ}(G, \mathcal{S})$ we need a generating set for $\operatorname{Aut}(\Gamma)$. This can be constructed as follows. In [3], Birman constructs a set of geometrically defined generators of $\mathcal{M}\left(T^{o}, \mathcal{S}\right)$ using Dehn twists and spin maps. The action of these geometric generators on $\pi_{1}\left(T^{o}, Q_{0}\right)$ can be written as substitution formulas in the generators $\left\langle\widetilde{\alpha}_{i}, \widetilde{\beta}_{i}, \tilde{\gamma}_{j} \mid 1 \leq i \leq \rho, 1 \leq j \leq r\right\rangle$ using the presentation (7) and then writing $\left\langle\alpha_{i}, \beta_{i}, \gamma_{j} \mid 1 \leq i \leq \rho, 1 \leq j \leq r\right\rangle$ as substitution formulas in the generators using the homomorphism (8) and the presentation (2). Any substitution formula can be tested against the presentation to see if it really is an automorphism. In Table 1, Table 2 and Table 3 we provide some examples, many of which first appeared in Harvey [20]. There are many more but we will not record them here since we are only going to use the much simpler transformations for abelian groups. 


\begin{tabular}{|c|c|c|c|c|}
\hline & $\alpha_{i}$ & $\beta_{i}$ & $\alpha_{i+1}$ & $\beta_{i+1}$ \\
\hline $\mathfrak{A}_{i}$ & $\alpha_{i}$ & $\beta_{i} \alpha_{i}$ & $\alpha_{i+1}$ & $\beta_{i+1}$ \\
\hline $\mathfrak{B}_{i}$ & $\alpha_{i} \beta_{i}$ & $\beta_{i}$ & $\alpha_{i+1}$ & $\beta_{i+1}$ \\
\hline $\mathfrak{R}_{i}$ & $\alpha_{i} \beta_{i} \alpha_{i}^{-1}$ & $\alpha_{i}^{-1}$ & $\alpha_{i+1}$ & $\beta_{i+1}$ \\
\hline $\mathfrak{S}_{i}$ & $\delta \alpha_{i+1} \delta^{-1}$ & $\delta \beta_{i+1} \delta^{-1}$ & $\alpha_{i}$ & $\beta_{i}$ \\
\hline $\mathfrak{Z}_{i}$ & $\alpha_{i} \alpha_{i+1}$ & $\alpha_{i+1}^{-1} \beta_{i} \alpha_{i+1}$ & $\epsilon \alpha_{i+1}$ & $\beta_{i+1} \beta_{i}^{-1} \epsilon^{-1}$ \\
\hline
\end{tabular}

Table 1: Action of automorphisms $\mathfrak{A}_{i}, \mathfrak{B}_{i}, \mathfrak{C}_{i}, \mathfrak{R}_{i}, \mathfrak{S}_{i}, \mathfrak{Z}_{i}$. Here $\delta=$ $\left[\alpha_{i}, \beta_{i}\right], \varepsilon=\left[\alpha_{i+1}^{-1}, \beta_{i}\right]$.

\begin{tabular}{|c|c|c|c|}
\hline & $\gamma_{j}$ & $\gamma_{j+1}$ & requirements \\
\hline $\mathfrak{T}_{j}$ & $\gamma_{j} \gamma_{j+1} \gamma_{j}^{-1}$ & $\gamma_{j}$ & $o\left(\gamma_{j}\right)=o\left(\gamma_{j+1}\right)$ \\
\hline $\mathfrak{T}_{j}^{-1}$ & $\gamma_{j+1}$ & $\gamma_{j+1}^{-1} \gamma_{j} \gamma_{j+1}$ & $o\left(\gamma_{j}\right)=o\left(\gamma_{j+1}\right)$ \\
\hline
\end{tabular}

Table 2: Action of $\mathfrak{T}_{j}$

\begin{tabular}{|c|c|c|c|c|}
\hline & $\alpha_{i}$ & $\beta_{i}$ & $\gamma_{j}$ & notes \\
\hline $\mathfrak{U}_{i, j}$ & $\alpha_{i}$ & $\beta_{i} x \gamma_{j} x^{-1}$ & $y \gamma_{j} y^{-1}$ & $x=\alpha_{i}^{-1} \beta_{i}^{-1} w_{2}, y=x^{-1} \alpha_{i}^{-1} x$ \\
\hline $\mathfrak{V}_{i, j}$ & $\alpha_{i} u \gamma_{j} u^{-1}$ & $\beta_{i}$ & $v \gamma_{j} v^{-1}$ & $u=\beta_{i} \alpha_{i}^{-1} \beta_{i}^{-1} w_{2}, u=v=u^{-1} \beta_{i} u$ \\
\hline
\end{tabular}

Table 3: Action of $\mathfrak{U}_{i, j}, \mathfrak{V}_{i, j}$. Here $w_{2}=\prod_{k=i+1}^{\rho}\left[\alpha_{k}, \beta_{k}\right] \prod_{k=1}^{j-1} \gamma_{k}$.

When the group $G$ is an additively written abelian group the formulas are much simpler since all commutators will disappear and elements are equal to their conjugates. To avoid confusing the additive formulas with the multiplicative formulas in the general case we will use uppercase letters for a generating vector viz., $\left(A_{1}, \ldots A_{\rho}, B_{1}, \ldots B_{\rho}, C_{1}, \ldots C_{\rho}\right)$. In this case an arbitrary assignment of elements of $G$ to the generators $\eta: \alpha_{i} \rightarrow A_{i}, \beta_{i} \rightarrow B_{i}$ will define an element of $\operatorname{Hom}(\Gamma, G)$ since the commutation relation is trivially satisfied. Our transformations above may be written

$$
\begin{aligned}
& \mathfrak{A}_{i}^{k}: B_{i} \rightarrow B_{i}+k A_{i} \\
& \mathfrak{B}_{i}^{k}: A_{i} \rightarrow A_{i}+k B_{i} \\
& \mathfrak{Z}_{i}^{k}: A_{i} \longrightarrow A_{i}+k A_{i+1}, B_{i+1} \longrightarrow B_{i+1}-k B_{i} \\
& \mathfrak{R}_{i}: A_{i} \rightarrow B_{i}, B_{i} \rightarrow-A_{i} \\
& \mathfrak{S}_{i}: A_{i} \rightarrow A_{i+1}, B_{i} \rightarrow B_{i+1}, A_{i+1} \rightarrow A_{i}, B_{i+1} \rightarrow B_{i}
\end{aligned}
$$


and trivial on all other generators. If we include branch points then we must also add an assignment $\eta: \gamma_{j} \rightarrow C_{j}$, with

$$
\begin{gathered}
o\left(C_{j}\right)=m_{j}, \\
C_{1}+C_{2}+\cdots+C_{r}=0 .
\end{gathered}
$$

We can then add to our table of transformations:

$$
\begin{aligned}
\mathfrak{T}_{i}: C_{j} & \rightarrow C_{j+1}, C_{j+1} \rightarrow C_{j}, m_{j}=m_{j+1} \\
\mathfrak{U}_{i, j}^{k}: B_{i} & \rightarrow B_{i}+k C_{j} \\
\mathfrak{V}_{i, j}^{k}: A_{i} & \rightarrow A_{i}+k C_{j}
\end{aligned}
$$

Obviously, any permutation of the $C_{j}$ preserving order is permissible.

When $G$ is abelian then the induced action of $\operatorname{Aut}(\Gamma)$ on a generating vector can be written in matrix vector form. Suppose that $\xi^{-1} \in \operatorname{Aut}(\Gamma)$ is induced by a homeomorphism of $T^{o}$ preserving branch order. Then

$$
\left(A_{1}, \ldots A_{\rho}, B_{1}, \ldots B_{\rho}, C_{1}, \ldots C_{r}\right) \longrightarrow\left(A_{1}, \ldots A_{\rho}, B_{1}, \ldots B_{\rho}, C_{1}, \ldots C_{r}\right) M
$$

where $M$ is a matrix of the form

$$
M=\left[\begin{array}{cc}
\mathrm{Sp} & Z \\
0 & P
\end{array}\right]
$$

where Sp is a $2 \rho \times 2 \rho$ integral symplectic matrix, $P$ is an $r \times r$ permutation matrix, and $Z$ is arbitrary integer valued matrix. The matrix $\mathrm{Sp}$ is the induced symplectic automorphism on $H_{1}(T ; \mathbb{Z})$. Perhaps the easiest way to see this is that each of the generators listed above has the given matrix decomposition (see also Gilman [16]).

\subsection{Hyperbolic-elliptic decomposition, abelian case}

In the group $\Gamma$ the generators $\left\langle\alpha_{i}, \beta_{i} \mid 1 \leq i \leq \rho\right\rangle$ are hyperbolic elements and the generators $\left\{\gamma_{j} \mid 1 \leq j \leq r\right\}$ are elliptic elements. Accordingly we define the hyperbolic (unramified) and elliptic (ramified) parts of $G: G^{h}=\left\langle A_{i}, B_{i} \mid 1 \leq i \leq \rho\right\rangle$ and $G^{e}=$ $\left\langle C_{j} \mid 1 \leq j \leq r\right\rangle$. We are interested in the action of $\operatorname{Aut}(\Gamma)$ on the splitting of $G$. Accordingly define for $\xi \in \operatorname{Aut}(\Gamma)$

$$
G^{h, \xi}=\left\langle\eta \circ \xi^{-1}\left(\alpha_{i}\right), \eta \circ \xi^{-1}\left(\beta_{i}\right) \mid 1 \leq i \leq \rho\right\rangle, \quad G^{e, \xi}=\left\langle\eta \circ \xi^{-1}\left(\gamma_{j}\right) \mid 1 \leq j \leq r\right\rangle .
$$

We have the following:

Proposition 2.4 Let $\eta: \alpha_{i} \rightarrow A_{i}, \beta_{i} \rightarrow B_{i}, \gamma_{j} \rightarrow C_{j}$, be an arbitrary assignment. Then $\eta$ extends to an epimorphism $\eta: \Gamma \rightarrow G$ if and only if the relations (9) and (10) hold and $G=\left\langle G^{h}, G^{e}\right\rangle$. 
Proposition 2.5 Let $\eta: \Gamma \rightarrow G$ be an epimorphism then $G^{e}=G^{e, \xi}$ for all $\xi \in \operatorname{Aut}(\Gamma)$.

Proof Let $\Gamma^{e}=\left\langle\gamma_{j} \mid 1 \leq j \leq r\right\rangle$. If $N\left(\Gamma^{e}\right)$ is the normal closure of $\Gamma^{e}$ then $G^{e}=$ $\eta\left(N\left(\Gamma^{e}\right)\right)$. It is well known that in $\Gamma$ every elliptic element is conjugate to one of the $\gamma_{j}$, and hence $\left\langle\eta \circ \xi^{-1}\left(C_{j}\right) \mid 1 \leq j \leq r\right\rangle=\eta\left(\xi^{-1}\left(N\left(\Gamma^{e}\right)\right)\right)=\eta\left(N\left(\Gamma^{e}\right)\right)=G^{e}$.

Proposition 2.6 The elliptic components $\left(C_{1}, \ldots C_{r}\right)$ and $\left(C_{1}^{\prime}, \ldots, C_{r}^{\prime}\right)$ of two abelian generating vectors are $\mathcal{M}(\Gamma)$-equivalent if and only if one is a permutation of the other: $C_{j}^{\prime}=C_{\pi j}$ for some permutation $\pi$.

Proof The automorphism $\xi$ is induced by a homeomorphism $h$ of $\left(T^{o}, Q_{0}\right)$ which must permute the punctures $Q_{1}, \ldots, Q_{r}$. Since $h$ is orientation-preserving then $h^{-1}\left(\widetilde{\gamma_{j}}\right)$ is a conjugate of some $\widetilde{\gamma_{\pi j}}$ for some permutation $\pi$.

Next let $G^{e}$ be a subgroup of $G$ and consider all possible subgroups $H$ such that $G=\left\langle H, G^{e}\right\rangle$. Let $H_{0}$ be one of smallest order. Then we may also assume that $G^{h}=H_{0}$.

Proposition 2.7 Let $\eta: \Gamma \rightarrow G$ be an action and let $H_{0}$ be the subgroup of smallest order such that $G=\left\langle H_{0}, G^{e}\right\rangle$. Then there is $\xi \in \mathcal{M}(\Gamma)$ such that $G^{h, \xi}=H_{0}$ and $G^{e, \xi}=G^{e}$.

Proof The equality $G^{e, \xi}=G^{e}$ is automatic. Each $A_{i}$ and $B_{i}$ may be written:

$$
\begin{aligned}
& A_{i}=A_{i}^{\prime}+a_{i, 1} C_{1}+\cdots+a_{i, r} C_{r}, A_{i}^{\prime} \in H_{0} \\
& B_{i}=B_{i}^{\prime}+b_{i, 1} C_{1}+\cdots+b_{i, r} C_{r}, B_{i}^{\prime} \in H_{0}
\end{aligned}
$$

By applying $\mathfrak{V}_{i, j}^{-a_{i, j}}$ we can remove the component $a_{i, j} C_{j}$ from the expression for $A_{i}$ and affect no other terms. Thus all the $A_{i}$ can be reduced to $A_{i}^{\prime}$. Similarly the $B_{i}$ can be reduced to $B_{i}^{\prime}$. Now $\left\langle A_{i}^{\prime}, B_{i}^{\prime} \mid 1 \leq i \leq \rho\right\rangle \subseteq H_{0}$ and $G=\left\langle\left\langle A_{i}^{\prime}, B_{i}^{\prime} \mid 1 \leq i \leq \rho\right\rangle, G^{e}\right\rangle$ by construction, so $H_{0}=\left\langle A_{i}^{\prime}, B_{i}^{\prime} \mid 1 \leq i \leq \rho\right\rangle$ by minimality.

\subsection{The elementary abelian case}

Finally let us assume that $G=\mathbb{F}_{p}^{w}$, an elementary abelian group considered as a vector space over $\mathbb{F}_{p}$. The generators $A_{i}, B_{i}, C_{j}$ may then be thought of as vectors over $\mathbb{F}_{p}$. Now let $X_{A B}$ be the matrix $\left[\begin{array}{llllll}A_{1} & \cdots & A_{\rho} & B_{1} & \cdots & B_{\rho}\end{array}\right]$ and $X_{C}$ be the matrix $\left[\begin{array}{lll}C_{1} & \cdots & C_{r}\end{array}\right]$. Then by Equation (11) the action of $(\omega, \xi)$ on $X=\left[\begin{array}{ll}X_{A B} & X_{C}\end{array}\right]$ is

$$
\left[\begin{array}{ll}
X_{A B} & X_{C}
\end{array}\right] \rightarrow M_{\omega}\left[\begin{array}{ll}
X_{A B} & X_{C}
\end{array}\right] M_{\xi}^{-1}
$$


where $M_{\omega} \in G L_{w}(p)$ and

$$
M_{\xi}^{-1}=\left[\begin{array}{cc}
\operatorname{Sp} & Z \\
0 & P
\end{array}\right]
$$

Next by Proposition 2.7 we assume that $\eta$ is chosen so that $\mathbb{F}_{p}^{w}=G^{h} \oplus G^{e}$. Now write $\mathbb{F}_{p}^{w}=\mathbb{F}_{p}^{u} \oplus \mathbb{F}_{p}^{v}$ and find an $N \in G L_{w}(p)$ such $N G^{h}=\mathbb{F}_{p}^{u} \oplus 0$ and $N G^{e}=0 \oplus \mathbb{F}_{p}^{v}$. It then follows that

$$
N\left[\begin{array}{ll}
X_{A B} & X_{C}
\end{array}\right]=\left[\begin{array}{cc}
Y_{A B} & 0 \\
0 & Y_{C}
\end{array}\right]
$$

where $Y_{A B}$ is $u \times 2 \rho$ matrix of rank $u$ and $Y_{C}$ is a $v \times r$ matrix of rank $v$. Thus we may assume the generating vector $X$ of our initial $\eta$ has the form of the right hand side of Equation (12). Now suppose that we look at a transformed generating vector $M_{\omega} X M_{\xi}^{-1}$ that has the same form ie:

$$
\begin{aligned}
{\left[\begin{array}{cc}
W_{A B} & 0 \\
0 & W_{C}
\end{array}\right] } & =\left[\begin{array}{ll}
M_{11} & M_{12} \\
M_{21} & M_{22}
\end{array}\right]\left[\begin{array}{cc}
Y_{A B} & 0 \\
0 & Y_{C}
\end{array}\right]\left[\begin{array}{cc}
S p & Z \\
0 & P
\end{array}\right] \\
& =\left[\begin{array}{ll}
M_{11} Y_{A B} \operatorname{Sp} & M_{11} Y_{A B} Z+M_{12} Y_{C} P \\
M_{21} Y_{A B} \operatorname{Sp} & M_{21} Y_{A B} Z+M_{22} Y_{C} P
\end{array}\right]
\end{aligned}
$$

Since $M_{21} Y_{A B} \mathrm{Sp}=0$ and $Y_{A B}$ Sp has rank $u$ then $M_{21}=0$, and $M_{11}$ and $M_{22}$ are invertible. Since $M_{11} Y_{A B} Z+M_{12} Y_{C} P$ is also assumed zero, it follows that

$$
\begin{aligned}
{\left[\begin{array}{cc}
W_{A B} & 0 \\
0 & W_{C}
\end{array}\right] } & =\left[\begin{array}{cc}
M_{11} Y_{A B} \mathrm{Sp} & 0 \\
0 & M_{22} Y_{C} P
\end{array}\right] \\
& =\left[\begin{array}{ll}
M_{11} & \\
& M_{22}
\end{array}\right]\left[\begin{array}{cc}
Y_{A B} & 0 \\
0 & Y_{C}
\end{array}\right]\left[\begin{array}{cc}
\mathrm{Sp} & \\
0 & P
\end{array}\right]
\end{aligned}
$$

ie, we can just find the equivalence classes of the unramified and the ramified components independently. We summarize the results in a theorem.

Theorem 2.8 Suppose that the group $G=\mathbb{F}_{p}^{w}$ acts on a surface $S$ with signature $\left(\rho ; p^{r}\right)$ and genus $1+p^{w}(\rho-1)+p^{w-1} \frac{r(p-1)}{2}$ determined by an epimorphism $\eta: \Gamma \rightarrow G$ (note that $r=1$ is infeasible). Then there are unique integers $u$, $v$ such that $w=u+v, 0 \leq u \leq 2 \rho, 1 \leq v<r$, a $u \times 2 \rho$ matrix $W_{A B}$ of rank $u$, and a $v \times r$ matrix $W_{C}$ of rank $v$ such that the matrix

$$
\left[\begin{array}{cc}
W_{A B} & 0 \\
0 & W_{C}
\end{array}\right]
$$

Algebraic $8 \mathcal{G}$ Geometric Topology, Volume 7 (2007) 
is a representative of the topological equivalence class of $G$-actions on $S$. Moreover if

$$
\left[\begin{array}{cc}
W_{A B}^{\prime} & 0 \\
0 & W_{C}^{\prime}
\end{array}\right]
$$

is another representative satisfying similar rank conditions for $W_{A B}^{\prime}$ and $W_{C}^{\prime}$ then $W_{A B}$ and $W_{A B}^{\prime}$ define equivalent unramified actions with signature $(\rho ;-)$ and $W_{C}$ and $W_{C}^{\prime}$ define equivalent purely ramified actions with signature $\left(0 ; p^{r}\right)$.

Corollary 2.9 For $0 \leq u \leq 2 \rho$, let $h_{u}$ be the number of equivalence classes of unramified actions of $\mathbb{F}_{p}^{u}$ on a surface of genus $1+p^{u}(\rho-1)$ with signature $(\rho ;-)$ and $h_{u}=0$ otherwise. Let $e_{v}$ be the number of equivalence classes of purely ramified actions of $\mathbb{F}_{p}^{v}$ on a surface $S$ of genus $1+p^{w-1} \frac{r(p-1)}{2}-p^{w}$ with signature $\left(0 ; p^{r}\right)$ for $1 \leq v<r$. Let $e_{0}=1$ and $e_{v}=0$ otherwise. Then the number of inequivalent actions of $\mathbb{F}_{p}^{w}$ on a surface of genus $1+p^{w}(\rho-1)+p^{w-1} \frac{r(p-1)}{2}$ with signature $\left(\rho ; p^{r}\right)$ is given by

$$
\text { \# actions }=\sum_{u=0}^{w} h_{u} e_{w-u} .
$$

\subsection{Cohomological invariants}

For abelian covers we may use cohomology to concoct an $\mathcal{M}$-invariant to distinguish classes. As noted above, for an abelian group $G, \operatorname{Hom}(\Gamma, G)$ classifies covers $T$ by subgroups of $G$. We have the sequence of equivalences

$$
\operatorname{Hom}(\Gamma, G) \bumpeq \operatorname{Hom}\left(H_{1}(T ; \mathbb{Z}), G\right) \bumpeq H^{1}(T ; G) .
$$

Given two elements of $\eta_{1}, \eta_{2} \in H^{1}(T ; G)$ we may consider the cup product $\eta_{1} \cup \eta_{2} \in$ $H^{1}(T ; G \otimes G)$. If $h$ is an orientation-preserving homeomorphism then

$$
h^{*} \eta_{1} \cup h^{*} \eta_{2}=h^{*}\left(\eta_{1} \cup \eta_{2}\right)=\eta_{1} \cup \eta_{2} .
$$

In particular if $\eta_{1}=\eta_{2}=\eta$ then

$$
h^{*} \eta \cup h^{*} \eta=\eta \cup \eta
$$

since $h$ is orientation preserving. It is not hard to show that if $\eta: \alpha_{i} \rightarrow A_{i}, \eta: \beta_{i} \rightarrow B_{i}$, then

$$
\eta \cup \eta=\sum_{i=1}^{\rho}\left(A_{i} \otimes B_{i}-B_{i} \otimes A_{i}\right)
$$

Observe that the invariance of $\eta \cup \eta$ under the transforms in Section 2.3 may also be proved by direct computation. 
Example 2.10 Let $G=C_{n} \oplus C_{m}$ with $x$ generating $C_{n}$ and $y$ generating $C_{m}$ additively (cyclic groups of order $m$ and $n$ ). Consider the following epimorphisms:

$$
\begin{aligned}
& \eta_{1}:\left(\alpha_{1}, \beta_{1}, \alpha_{2}, \beta_{2}\right) \rightarrow(x, y, 0,0) \\
& \eta_{2}:\left(\alpha_{1}, \beta_{1}, \alpha_{2}, \beta_{2}\right) \rightarrow(x, 0, y, 0)
\end{aligned}
$$

Then

$$
\begin{aligned}
& \eta_{1} \cup \eta_{1}=x \otimes y-y \otimes x \\
& \eta_{2} \cup \eta_{2}=0
\end{aligned}
$$

and so the epimorphisms are different under the action of Aut $(\Gamma(2 ;-))$.

\section{The unramified abelian case}

In this section, we consider the case when $G$ is abelian and $\Gamma=\Pi_{\rho}$ is torsion-free. For the special case when $G$ is elementary abelian, complete results were derived in Broughton [9]. We shall apply the ideas from this case to the more general abelian case. Due to the complexity of the problem, we shall only produce partial results, though the results we derive will be sufficient to produce explicit results for genus up to 65 . We shall also illustrate how in principle one could use the results to classify all fixed point abelian actions for arbitrary genus. It should be noted that we do not consider the abelian case for general $\Gamma$ because Theorem 2.8 no longer holds so there could be overlap between $G^{h}$ and $G^{e}$ making the problem much more difficult.

\subsection{The unramified elementary abelian case}

The following was proved in Broughton [9] and completely classifies all unramified elementary abelian actions up to topological equivalence (to keep notation consistent with the elementary abelian case, for an abelian group $G$, by $p$-rank we mean the number of invariant factors of $G$ ). The corollary immediately follows.

Theorem 3.1 Suppose $\Gamma=\Pi_{\rho}$ is a surface group of orbit genus $\rho$ and generators $\alpha_{1}, \ldots, \alpha_{\rho}, \beta_{1}, \ldots b_{\rho}$ and $G$ is an elementary abelian group of $p$-rank $r \leq 2 \rho$ with generators $\omega_{1}, \ldots, \omega_{r}$. Then there exists an integer $r / 2 \leq K \leq \min (\rho, r)$ such that any epimorphism from $\Gamma$ onto $G$ is Aut $(G) \times \operatorname{Aut}(\Gamma)$-equivalent to one of those below:

$$
\eta:= \begin{cases}\eta\left(\alpha_{i}\right)=\omega_{i} & i \leq K \\ \eta\left(\alpha_{i}\right)=0 & i>K \\ \eta\left(\beta_{i}\right)=\omega_{i+K} & i \leq r-K \\ \eta\left(\beta_{i}\right)=0 & i>r-K\end{cases}
$$

Algebraic 83 Geometric Topology, Volume 7 (2007) 
Corollary 3.2 Suppose $G$ is an elementary abelian group of $p$-rank $r$, let $\mathcal{M}_{\sigma}$ denote the mapping class group of a closed surface of genus $\sigma$ and let $\rho=\left(\sigma-1+p^{r}\right) / p^{r}$. If $\rho$ is not an integer, there are no conjugacy classes of subgroups of $\mathcal{M}_{\sigma}$ isomorphic to $G$ with fixed point free action. Else, the number of conjugacy classes of subgroups of $\mathcal{M}_{\sigma}$ isomorphic to $G$ with fixed point free action is calculated as follows: if $r \leq \rho$, there are $r / 2+1$ different classes of epimorphisms for $r$ even and $(r+1) / 2$ epimorphisms if $r$ is odd, if $r=\rho+i$ with $0<i<\rho$ there are $(\rho-i) / 2$ classes of epimorphisms if $\rho-i$ is even and $(\rho-i+1) / 2$ if $\rho-i$ is odd, and if $r=2 \rho$ or $r=1$, there is just one class.

We illustrate with an example.

Example 3.3 Suppose $\Gamma$ has genus 2 and $G$ is an elementary abelian $p$-group of $p$-rank 2. If $\eta: \Gamma \rightarrow G$ is a surface kernel epimorphism, then using the RiemannHurwitz formula, the genus $\sigma$ of the kernel will be $\sigma=p^{2}+1$. In fact, by simple application of the Riemann Hurwitz formula, it can be shown that for $p>5, \Gamma$ is the only Fuchsian group which admits an elementary abelian quotient of order $p^{2}$ with kernel of orbit genus $p^{2}+1$ (for $p=5$, see Example 5.1). This means for $p>5$, in $\mathcal{M}_{p^{2}+1}$, the conjugacy classes of elementary abelian subgroups of order $p^{2}$ will be in a one to one correspondence with the classes of epimorphisms from $\Gamma$ onto $G$. Applying Corollary 3.2, there are two such classes.

\subsection{The general unramified abelian case}

We now consider partial results for the general abelian case. Before we start, we introduce some notation and terminology.

Notation For the rest of this section, $G$ will denote an additively written abelian group of $p$-rank $r$ with invariant factors $n_{1} \geq n_{2} \geq \cdots \geq n_{r}$ where $n_{i+1} \mid n_{i}$ and $\omega_{1}, \ldots, \omega_{r}$ are a fixed set of generators of orders $n_{1}, \ldots, n_{r}$ respectively. Also, for an integer $n$, $C_{n}$ denotes the cyclic group of order $n$.

The following two steps can be taken to classify all fixed point free $G$-actions on a surface: first determine a set of epimorphisms with the property that every epimorphism is equivalent to one in this set, and then reduce this set so no two epimorphisms are equivalent. In the following, we shall consider the first step. Though in general we shall not consider the problem of distinguishing between classes, we shall present explicit examples showing how in principle one could tackle this problem. Note that $r \leq 2 \rho$, so we only need consider epimorphisms from $\Gamma$ to $G$ with $p$-rank at most $2 \rho$.

Algebraic 83 Geometric Topology, Volume 7 (2007) 
Proposition 3.4 Any epimorphism $\eta: \Gamma \rightarrow G$ is equivalent to one of those described below:

(1) $r \leq \rho$.

$$
\eta:= \begin{cases}\eta\left(\alpha_{i}\right)=\omega_{i} & i \leq r \\ \eta\left(\alpha_{i}\right)=0 & i>r \\ \eta\left(\beta_{i}\right)=\sum_{j=i+1}^{r} N_{\beta_{i}, \omega_{j}} \omega_{j} & i \leq r \\ \eta\left(\beta_{i}\right)=0 & i>r\end{cases}
$$

where

- $\left(n_{i+1}, N_{\beta_{i}, \omega_{i+1}}\right)>1$ or $N_{\beta_{i}, \omega_{i+1}}=1$ for each $i \leq r-1$

- for each $i$, if $j>i+1$ is the largest integer such that $N_{\beta_{i}, \omega_{j}} \neq 0$, then $\left\langle N_{\beta_{i}, \omega_{j}} \omega_{j}\right\rangle \leq\left\langle N_{\beta_{i+1}, \omega_{j}} \omega_{j}\right\rangle$.

(2) $r>\rho$.

$$
\eta:= \begin{cases}\eta\left(\alpha_{i}\right)=\omega_{i} & 1 \leq i \leq \rho \\ \eta\left(\beta_{i}\right)=\sum_{j=i+1}^{r} N_{\beta_{i}, \omega_{j}} \omega_{j}, & 1 \leq i \leq 2 \rho-r \\ \eta\left(\beta_{i}\right)=\omega_{2 \rho-i+1}+\sum_{j=i+1}^{2 \rho-i} N_{\beta_{i}, \omega_{j}} \omega_{j} & 2 \rho-r<i<\rho \\ \eta\left(\beta_{\rho}\right)=\omega_{\rho+1} & i=\rho\end{cases}
$$

where

- for $i>2 \rho-r$ either the order of $N_{\beta_{i}, \omega_{j}} \omega_{j}$ does not divide $n_{r}$ or $N_{\beta_{i}, \omega_{j}}=0$

- for $i \leq 2 \rho-r$ if $j>i+1$ is the largest integer such that $N_{\beta_{i}, \omega_{j}} \neq 0$, then $\left\langle N_{\beta_{i}, \omega_{j}} \omega_{j}\right\rangle \leq\left\langle N_{\beta_{i+1}, \omega_{j}} \omega_{j}\right\rangle$

- for $i \leq 2 \rho-r-1,\left(n_{i}, N_{\beta_{i}, \omega_{i+1}}\right)>1$ or $N_{\beta_{i}, \omega_{i+1}}=1$.

Proof We shall use induction on the $p$-rank of the group $G$. For $p$-rank $r=1$, we are done by Harvey [21, Theorem 14]. Assuming the result holds for $p$-rank $r-1$, we shall prove it holds for $p$-rank $r$. The proof falls into two different cases depending upon whether $1<r \leq \rho$ or $\rho<r \leq 2 \rho$. First suppose that $r \leq \rho$.

Let $\eta: \Gamma \rightarrow G=C_{n_{1}} \times \cdots \times C_{n_{r}}$ denote the epimorphism onto $G$ and $\Phi: G \rightarrow$ $C_{n_{1}} \times \cdots \times C_{n_{r-1}}$ the projection map onto the quotient group $G / C_{n_{r}}$ (by abuse of notation, we identify $C_{n_{1}} \times \cdots \times C_{n_{r-1}}$ with the corresponding subgroup of $G$ ). Since $\Gamma$ is torsion-free, all subgroups will be torsion-free. In particular, the map $\Phi \circ \eta$ will be a surface kernel epimorphism from $\Gamma$ onto $C_{n_{1}} \times \cdots \times C_{n_{r-1}}$, so by induction, will be equivalent to one as given in the statement of the proposition. Lifting to $G$ and 
composing with appropriate automorphisms of $G$, it follows that $\eta$ is equivalent to an epimorphism of the following form:

$$
\eta:= \begin{cases}\eta\left(\alpha_{i}\right)=\omega_{i} & i<r \\ \eta\left(\alpha_{i}\right)=m_{i} \omega_{r} & i \geq r \\ \eta\left(\beta_{i}\right)=M_{i} \omega_{r}+\sum_{j=i+1}^{r-1} N_{\beta_{i}, \omega_{j}} \omega_{j} & i \leq r \\ \eta\left(\beta_{i}\right)=M_{i} \omega_{r} & i>r\end{cases}
$$

for integers $m_{i}, r \leq i \leq \rho$ and $M_{j}, 1 \leq j \leq \rho$ (for convenience, by abuse of notation, we shall denote any epimorphism equivalent to $\eta$ by $\eta$ ). Similar to the elementary abelian case in Broughton [9], we now reduce using the automorphisms from Aut $(\Gamma)$ developed in Section 2.3 and the automorphisms of the abelian group $G$. In most instances, since the reduction steps are between pairs of pairs of generators and constant on all others, we shall just consider the pairs which are changed. To start, let $k$ be the smallest integer such that $\left(M_{2}-k M_{1}\right) \omega_{r}$ generates the subgroup generated by $M_{1} \omega_{r}$ and $M_{2} \omega_{r}$ and let $\Phi \in \operatorname{Aut}(G)$ where $\Phi\left(\omega_{1}\right)=\omega_{1}+k \omega_{2}$ and the identity map on all other generators. Then the map $\mathfrak{A}_{1}^{n_{2}-N_{\beta_{1}, \omega_{2}}} \Phi \circ \mathfrak{Z}_{1}^{k}$ modifies the images of $\alpha_{1}, \alpha_{2}$, $\beta_{1}$ and $\beta_{2}$ but acts trivially on the images of $\alpha_{i}$ and $\beta_{i}$ for $i \geq 3$. Specifically, after renaming the coefficients, we get:

$$
\begin{aligned}
\left(\alpha_{1}, \beta_{1}, \alpha_{2}, \beta_{2}\right) & \longrightarrow\left(\omega_{1}, M_{1} \omega_{r}+\sum_{j=2}^{r-1} N_{\beta_{i}, \omega_{j}} \omega_{j}, \omega_{2}, M_{2} \omega_{r}+\sum_{j=3}^{r-1} N_{\beta_{i}, \omega_{j}} \omega_{j}\right) \\
& \longrightarrow\left(\omega_{1}, \sum_{j=2}^{r} N_{\beta_{1}, \omega_{i}} \omega_{i}, \omega_{2}, \sum_{j=3}^{r} N_{\beta_{2}, \omega_{i}} \omega_{i}\right)
\end{aligned}
$$

where $\left\langle N_{\beta_{1}, r} \omega_{r}\right\rangle \leq\left\langle N_{\beta_{2}, r} \omega_{r}\right\rangle$. Applying similar transformations to all proceeding pairs of pairs up to the pair $\left(\alpha_{r}, \beta_{r}\right)$ we get:

$$
\eta:= \begin{cases}\eta\left(\alpha_{i}\right)=\omega_{i} & i<r \\ \eta\left(\alpha_{i}\right)=m_{i} \omega_{r} & i \geq r \\ \eta\left(\beta_{i}\right)=\sum_{j=i+1}^{r} N_{\beta_{i}, \omega_{j}} \omega_{j} & i \leq r \\ \eta\left(\beta_{i}\right)=M_{i} \omega_{r} & i>r\end{cases}
$$

where $\left\langle N_{\beta_{i}, r} \omega_{r}\right\rangle \leq\left\langle N_{\beta_{i+1}, r} \omega_{r}\right\rangle$ for all $i$. In particular, this implies

$$
\left\langle N_{\beta_{1}, r}, N_{\beta_{2}, r}, \ldots, N_{\beta_{r-1}, r} \omega_{r}\right\rangle \leq\left\langle N_{\beta_{r}, r} \omega_{r}\right\rangle .
$$


Next, we reduce the pairs $\left(\alpha_{i}, \beta_{i}\right)$ with $i \geq r$. First, to any such pair, we apply $\mathfrak{B}_{i}^{k}$ where $k$ is the smallest integer such that $\left(m_{i}+k M_{i}\right) \omega_{r}$ generates the subgroup generated by $m_{i} \omega_{r}$ and $M_{i} \omega_{r}$ (for brevity, by abuse of notation, we rename $\left(m_{i}+k M_{i}\right)$ by $m_{i}$ ). Then for a given $i$, since $\left\langle M_{i} \omega_{r}\right\rangle \leq\left\langle m_{i} \omega_{r}\right\rangle$, there exists $n$ such that $\left(M_{i}+n m_{i}\right) \omega_{r}=0$ Applying the automorphism $\mathfrak{A}_{i}^{n}$, we get $\left(\alpha_{i}, \beta_{i}\right) \rightarrow\left(0, M_{i} \omega_{r}\right)$. Following this, for the pairs, $\left(\alpha_{\rho-1}, \beta_{\rho-1}, \alpha_{\rho}, \beta_{\rho}\right)$, we apply $\mathfrak{A}_{\rho-1}^{k}$ where $k$ is the smallest integer such that $\left(M_{\rho}-k M_{\rho-1}\right) \omega_{r}$ generates the subgroup generated by $M_{\rho} \omega_{r}$ and $M_{\rho-1} \omega_{r}$. Renaming $\left(M_{\rho}-k M_{\rho-1}\right) \omega_{r}$ by $M_{\rho}$, we get $\left(\alpha_{\rho-1}, \beta_{\rho-1}, \alpha_{\rho}, \beta_{\rho}\right) \rightarrow\left(M_{\rho-1} \omega_{r}, 0, M_{\rho} \omega_{r}, 0\right)$ where $\left\langle M_{\rho-1} \omega_{r}\right\rangle \leq\left\langle M_{\rho} \omega_{r}\right\rangle$. Finally, we eliminate the image of $\beta_{\rho-1}$ by applying $\mathfrak{A}_{\rho-1}^{n}$ where $n$ is the smallest integer such that $\left(M_{\rho-1}-n M_{\rho}\right) \omega_{r}=0$ and then apply $\mathfrak{S}_{\rho-1}$ and $\mathfrak{R}_{\rho-1}$ giving $\left(\alpha_{\rho-1}, \beta_{\rho-1}, \alpha_{\rho}, \beta_{\rho}\right) \rightarrow\left(0,0, M_{\rho-1} \omega_{r}, 0\right)$ (after renaming coefficients). Applying similar transformations to pairs $\left(\alpha_{i}, \beta_{i}, \alpha_{i+1}, \beta_{i+1}\right)$ with $i>r$, we get:

$$
\eta:= \begin{cases}\eta\left(\alpha_{i}\right)=\omega_{i} & i<r \\ \eta\left(\alpha_{r}\right)=M \omega_{r} & i=r \\ \eta\left(\alpha_{i}\right)=0 & i>r \\ \eta\left(\beta_{i}\right)=\sum_{j=i+1}^{r} N_{\beta_{i}, \omega_{j}} \omega_{j} & i \leq r \\ \eta\left(\beta_{i}\right)=0 & i>r\end{cases}
$$

for some integer $M$. Finally, we apply $\mathfrak{Z}_{r-1}^{k}$ where $k$ is the smallest integer such that $\left(M-k N_{\beta_{r-1}, \omega_{r}}, n_{r}\right)=1$ (note that such an integer exists since $M \omega_{r}$ and $N_{\beta_{r-1}, \omega_{r}} \omega_{r}$ generate $\left\langle\omega_{r}\right\rangle$ ). Finally, by applying $\mathfrak{G}_{r}$ and an appropriate automorphism of $G$, after renaming the coefficients, we get:

$$
\eta:= \begin{cases}\eta\left(\alpha_{i}\right)=\omega_{i} & i \leq r \\ \eta\left(\alpha_{i}\right)=0 & i>r \\ \eta\left(\beta_{i}\right)=\sum_{j=i+1}^{r} N_{\beta_{i}, \omega_{j}} \omega_{j} & i \leq r \\ \eta\left(\beta_{i}\right)=0 & i>r\end{cases}
$$

For the last reduction step, if $\left(N_{\beta_{r-1}, \omega_{r}}, n_{r}\right)=1$, we first apply $\Phi \in \operatorname{Aut}(G)$ such that $\Phi\left(N_{\beta_{r-1}, \omega_{r}} \omega_{r}\right)=\omega_{r}$ and identity on all other generators. Then, assuming $b$ is the integer with $\Phi\left(\omega_{r}\right)=b \omega_{r}$, we perform $\mathfrak{G}_{r} \circ \mathfrak{Z}_{r-1}^{b+1} \circ \mathfrak{G}_{r} \circ \Phi$ giving $\left(\alpha_{r-1}, \beta_{r-1}, \alpha_{r}, \beta_{r}\right) \rightarrow$ $\left(\omega_{r-1}, \omega_{r}, \omega_{r}, 0\right)$. Note that for $l<r$, none of the transformations used during this proof change the fact that $\left(n_{l}, N_{\beta_{l-1}, \omega_{l}}\right)>1$ or $N_{\beta_{l-1}, \omega_{l}}=1$. Observe also that the reduction methods used imply that if $j>i+1$ is the largest integer such that $N_{\beta_{i}, \omega_{j}} \neq 0$, then $\left\langle N_{\beta_{i}, \omega_{j}} \omega_{j}\right\rangle \leq\left\langle N_{\beta_{i+1}, \omega_{j}} \omega_{j}\right\rangle$. 
Now suppose that $r>\rho$. Since the arguments regarding transformations are similar to those used for the previous case, we shall skip many steps. Assuming the result holds when the $p$-rank of the group is $r-1 \geq \rho$, induction implies after composing with appropriate automorphisms of $G, \eta$ has the following form:

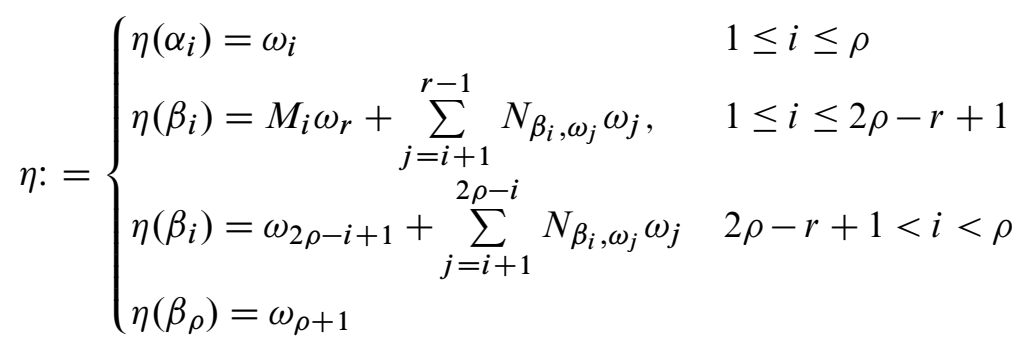

As with the previous case, for each $i<r$, we can use the transformations $\mathfrak{Z}_{i}, \mathfrak{A}_{i}$ and appropriate automorphisms of $G$ to move a generator for $\left\langle\omega_{r}\right\rangle$ to the image of $\beta_{2 \rho-r+1}$ giving

$$
\eta\left(\beta_{2 \rho-r+1}\right)=\omega_{2 \rho-r+1}+\sum_{j=r+1}^{2 \rho-r} N_{\beta_{i}, \omega_{j}} \omega_{j} .
$$

Applying these transformations, we get:

$$
\eta:= \begin{cases}\eta\left(\alpha_{i}\right)=\omega_{i} & 1 \leq i \leq \rho \\ \eta\left(\beta_{i}\right)=\sum_{j=i+1}^{r} N_{\beta_{i}, \omega_{j}} \omega_{j}, & 1 \leq i \leq 2 \rho-r \\ \eta\left(\beta_{i}\right)=\omega_{2 \rho-i+1}+\sum_{j=i+1}^{2 \rho-i} N_{\beta_{i}, \omega_{j}} \omega_{j} & 2 \rho-r+1 \leq i<\rho \\ \eta\left(\beta_{\rho}\right)=\omega_{\rho+1} & \end{cases}
$$

where $\left\langle N_{\beta_{1}, \omega_{r}} \omega_{r}\right\rangle \leq\left\langle N_{\beta_{2}, \omega_{j}} \omega_{j}\right\rangle \leq \cdots \leq\left\langle N_{\beta_{2 \rho-r+1}, \omega_{r}} \omega_{r}\right\rangle$.

For the last reduction step, we apply an appropriate automorphism of $G$ that acts trivially on all generators except $\omega_{r}$ and eliminates all elements from the sum $\sum_{j=i+1}^{r-1} N_{\beta_{i}, \omega_{j}} \omega_{j}$ with the order of $N_{\beta_{i}, \omega_{j}} \omega_{j}$ dividing $n_{r}$. Then we get:

$$
\eta:= \begin{cases}\eta\left(\alpha_{i}\right)=\omega_{i} & 1 \leq i \leq \rho \\ \eta\left(\beta_{i}\right)=\sum_{j=i+1}^{r} N_{\beta_{i}, \omega_{j}} \omega_{j}, & 1 \leq i \leq 2 \rho-r \\ \eta\left(\beta_{i}\right)=\omega_{2 \rho-i+1}+\sum_{j=i+1}^{2 \rho-i} N_{\beta_{i}, \omega_{j}} \omega_{j} & 2 \rho-r<i\end{cases}
$$

Algebraic $\mathcal{E}$ Geometric Topology, Volume 7 (2007) 
where the order of $N_{\beta_{i}, \omega_{j}} \omega_{j}$ does not divide $n_{r}$ for $i>2 \rho-r$. As with the last case, if $i \leq 2 \rho-r$, the reduction methods used implies that if $j>i+1$ is the largest integer such that $N_{\beta_{i}, \omega_{j}} \neq 0$, then $\left\langle N_{\beta_{i}, \omega_{j}} \omega_{j}\right\rangle \leq\left\langle N_{\beta_{i+1}, \omega_{j}} \omega_{j}\right\rangle$.

We note that though more refined sets of epimorphisms can be obtained (in particular, the elementary abelian case), our goal was to provide a set of epimorphisms independent of the genus and invariant factors. We finish with an explicit example showing how to use Proposition 3.4 to determine all classes of fixed point free actions.

Example 3.5 Suppose that $\Gamma$ has genus 2 and $G$ has $p$-rank 3 with invariant factors 4, 4, 2. Proposition 3.4 implies there are up to six classes of epimorphisms of the form $\left(\alpha_{1}, \beta_{1}, \alpha_{2}, \beta_{2}\right) \rightarrow\left(\omega_{1}, a \omega_{2}+b \omega_{3}, \omega_{2}, \omega_{3}\right)$ where $a=0,1,2$ and $b=0,1$. Using elements of Aut $(G) \times \operatorname{Aut}(\Gamma)$ it is fairly straight forward to reduce each of these epimorphisms to one of the following three:

$$
\begin{aligned}
& \eta_{1}:=\left(\alpha_{1}, \beta_{1}, \alpha_{2}, \beta_{2}\right) \rightarrow\left(\omega_{1}, \omega_{2}, \omega_{3}, 0\right) \\
& \eta_{2}:=\left(\alpha_{1}, \beta_{1}, \alpha_{2}, \beta_{2}\right) \rightarrow\left(\omega_{1}, \omega_{3}, \omega_{2}, 0\right) \\
& \eta_{3}:=\left(\alpha_{1}, \beta_{1}, \alpha_{2}, \beta_{2}\right) \rightarrow\left(\omega_{1}, \omega_{2}, 2 \omega_{2}, \omega_{3}\right)
\end{aligned}
$$

We shall show that these three epimorphisms are distinct.

First note that if $\eta_{i}$ is equivalent to $\eta_{j}$, then there exists $\theta \in \operatorname{Aut}(G)$ and $\zeta \in \operatorname{Aut}(\Gamma)$ such that $\theta \circ \eta_{i} \circ \zeta=\eta_{j}$. Since the cup product $\cup$ is an Aut $(\Gamma)$ invariant, it follows that $\left(\theta \circ \eta_{i}\right) \cup\left(\theta \circ \eta_{i}\right)=\eta_{j} \cup \eta_{j}$. Therefore, in order to show distinctness, it suffices to show that for $i \neq j$, there does not exist $\theta \in \operatorname{Aut}(G)$ such that $\left(\theta \circ \eta_{i}\right) \cup\left(\theta \circ \eta_{i}\right)=\eta_{j} \cup \eta_{j}$. Since $\operatorname{Aut}(G)$ is finite, this can be checked directly. We illustrate by showing that $\eta_{1}$ and $\eta_{2}$ are not equivalent.

First, taking the cup product we get

and

$$
\eta_{1} \cup \eta_{1}=\omega_{1} \otimes \omega_{2}-\omega_{2} \otimes \omega_{1}
$$

$$
\theta \circ \eta_{2} \cup \theta \circ \eta_{2}=\theta\left(\omega_{1}\right) \otimes \theta\left(\omega_{3}\right)-\theta\left(\omega_{3}\right) \otimes \theta\left(\omega_{1}\right) \text {. }
$$

Assuming that $\rho\left(\omega_{1}\right)=A \omega_{1}+B \omega_{2}+C \omega_{3}$ and $\rho\left(\omega_{3}\right)=D \omega_{1}+E \omega_{2}+F \omega_{3}$, by simplifying the tensor product and combining like terms, in order for these products to be equal, we must have $(A E-D B)=1 \bmod (4)$. However, this is impossible since both $D$ and $E$ must be divisible by 2 (since $\omega_{3}$ has order 2). Thus $\eta_{1}$ and $\eta_{2}$ are not equivalent. All other cases follow similar arguments and so it follows that there are three topological equivalence classes of fixed point $G$-actions on a surface of genus 33 . 


\subsection{Classification for Genera $\leq 65$}

Due to the ad hoc style arguments which seem required to distinguish classes, we fall short of a general classification for abelian groups. However, Proposition 3.4 coupled with the following results will allow a classification up to genus 65 (and in fact is enough to classify certain infinite families).

Lemma 3.6 If $G$ has $p$-rank 2 and $n_{2}$ is square free, then for each divisor $N$ of $n_{2}$ with $1 \leq N \leq n_{2}$, any epimorphism $\eta: \Gamma \rightarrow G$ is equivalent to one of those described below:

$$
\eta:= \begin{cases}\eta\left(\alpha_{1}\right)=\omega_{1} & \\ \eta\left(\alpha_{2}\right)=\omega_{2} & \\ \eta\left(\alpha_{i}\right)=0 & i>2 \\ \eta\left(\beta_{1}\right)=N \omega_{2} & \\ \eta\left(\beta_{i}\right)=0 & i>1\end{cases}
$$

Moreover, no two such epimorphisms are equivalent under the action of Aut $(G) \times$ Aut $(\Gamma)$, so in particular, there are $d\left(n_{2}\right)$ epimorphisms up to the action of Aut $(G) \times$ Aut $(\Gamma)$ (where $d\left(n_{2}\right)$ denotes the number of divisors of $n_{2}$ ).

Proof By Proposition 3.4, any epimorphism from $\Gamma$ onto a $p$-rank 2 abelian group will be equivalent to one of the form $\eta_{N}:\left(\alpha_{1}, \beta_{1}, \alpha_{2}, \beta_{2}\right) \rightarrow\left(\omega_{1}, N \omega_{2}, \omega_{2}, 0\right)$ with the image of all other generators being trivial. Therefore, we just need to determine when $\eta_{N}$ and $\eta_{M}$ are equivalent for $M \neq N$. We start by showing that if $\operatorname{gcd}(n, N) \neq$ $\operatorname{gcd}(n, M)$, then $\eta_{N}$ and $\eta_{M}$ are not equivalent.

First, using the arguments from Example 3.5, it suffices to show that there does not exist $\theta \in \operatorname{Aut}(G)$ such that $\left(\theta \circ \eta_{N}\right) \cup\left(\theta \circ \eta_{N}\right)=\eta_{M} \cup \eta_{M}$. In general, if $\theta \in \operatorname{Aut}(G)$, then $\theta$ can be identified with a $2 \times 2$ matrix with integer coefficients with the action of $\theta$ on the generators $\omega_{1}$ and $\omega_{2}$ defined as follows:

$$
\theta \cdot\left|\begin{array}{l}
\omega_{1} \\
\omega_{2}
\end{array}\right|=\left|\begin{array}{ll}
A & B \\
C & D
\end{array}\right| \cdot\left|\begin{array}{l}
\omega_{1} \\
\omega_{2}
\end{array}\right|=\left|\begin{array}{l}
A \omega_{1}+B \omega_{2} \\
C \omega_{1}+B \omega_{2}
\end{array}\right|
$$

Under such a realization, since this map must restrict to an automorphism of the subgroup $C_{n_{2}} \times C_{n_{2}}$, the determinant $\operatorname{det}(\rho)=A D-B C$ must be a unit in $C_{n_{2}}$. Assuming $\theta$ has this form, calculating the two cup products and simplifying, we get

and

$$
\left.\left(\theta \circ \eta_{N}\right) \cup\left(\theta \circ \eta_{N}\right)=N \operatorname{det}(\theta)\left(\omega_{1} \otimes \omega_{2}-\omega_{2} \otimes \omega_{1}\right)\right)
$$

$$
\eta_{M} \cup \eta_{M}=M\left(\omega_{1} \otimes \omega_{2}-\omega_{2} \otimes \omega_{1}\right) \text {. }
$$


Since $\operatorname{det}(\theta)$ is a unit in $C_{n_{2}}$, if $\operatorname{gcd}(n, N) \neq \operatorname{gcd}(n, M)$, then we can never have $N \operatorname{det}(\theta)=M$ (else they would be associates in the ring $C_{n_{2}}$ and thus we would have $\operatorname{gcd}(n, N)=\operatorname{gcd}(n, M))$. Thus if $\operatorname{gcd}(n, N) \neq \operatorname{gcd}(n, M)$ then $\eta_{N}$ and $\eta_{M}$ define inequivalent classes. To finish, we need to show if $\operatorname{gcd}(n, N)=\operatorname{gcd}(n, M)$, then $\eta_{N}$ and $\eta_{M}$ are equivalent.

Suppose $n=p_{1} \ldots p_{s} q_{1} \ldots q_{r}$ where the $p_{i}$ and $q_{i}$ are all distinct primes and $d=$ $p_{1} \ldots p_{s}=\operatorname{gcd}(n, N)$. Observe that by applying $\mathfrak{S}_{2}$ followed by $\mathfrak{Z}_{1}^{k}$ for any integer $k$ to $\eta_{N}$, we have the epimorphism $\left(\alpha_{1}, \beta_{1}, \alpha_{2}, \beta_{2}\right) \rightarrow\left(\omega_{1}, N \omega_{2}, 0,(1-k N) \omega_{2}\right)$. Moreover, if $\left(1-k N, n_{2}\right)=1$, then there exists an integer $a$ which induces an automorphism of $C_{n_{2}}$ via multiplication, such that $a(1-k N)=1 \bmod \left(n_{2}\right)$. Applying this automorphism and reapplying $\mathfrak{S}_{2}$, we see that $\eta_{N}$ is equivalent to $\eta_{a N}$ for any such $a$ and $\left(n_{2}, a N\right)=d$ (since $a$ is a unit in $\left.C_{n_{2}}\right)$.

Assume $\left((1-k N), n_{2}\right)=1$ and $\left((1-c N), n_{2}\right)=1$, let $a=(1-k N)^{-1}$ and $b=(1-c N)^{-1}$ (all taken $\left.\bmod \left(n_{2}\right)\right)$. In order to avoid redundancy, we may assume $0 \leq k, c \leq q_{1} \ldots q_{r}$. Now if $a N=b N \bmod \left(n_{2}\right)$, then $(1-k N)=(1-c N)$ and consequently $q_{1} \ldots q_{r} \mid(k-c)$. However, this implies $k=c+a q_{1} \ldots q_{r}$ which contradicts that $0 \leq k, c \leq q_{1} \ldots q_{r}$ unless $a=0$. Thus $\eta_{a N}=\eta_{b N}$ only when $a=b$. Therefore, since there are precisely $\phi\left(n_{2} / d\right)=\left(q_{1}-1\right)\left(q_{2}-1\right) \ldots\left(q_{r}-1\right)$ different integers $M$ with $\left(n_{2}, M\right)=d$, it suffices to show that there are precisely $\left(q_{1}-1\right)\left(q_{2}-1\right) \ldots\left(q_{r}-1\right)$ choices for $k$ such that $\left(1-k N, n_{2}\right)=1$.

Observe that $p_{i}$ does not divide $1-k N$ for any value of $i$ or $k$, so it follows that $\left(1-k N, n_{2}\right)=1$ provided $T=q_{i_{1}} \ldots q_{i_{t}}$ does not divide $1-k N$ for any divisor $T$ of $q_{1} \ldots q_{r}$. Suppose $T=q_{i_{1}} \ldots q_{i_{t}}$ is a divisor of $q_{1} \ldots q_{r}$. Then $(T, N)=1$ (since $\left.\operatorname{gcd}\left(n_{2}, N\right)=d\right)$, so there exists $k$ and $c$ such that $c T+k N=1$, or $c T=1-k N$. Moreover, if $c_{1} T+k_{1} N=1$ for any other $c_{1}$ and $k_{1}$, we must have $c_{1}=c+v N$ for some integer $v$ and for every integer $v$, if we define $c_{1}=c+v N$ and $k_{1}=k-T v$, then $c_{1} T=1-k_{1} N$. This implies there will be precisely $q_{1} \ldots q_{r} / T$ values of $k$ for which $T$ divides $1-k N$. Since there are $q_{1} \ldots q_{r}$ total choices for $k$ and $q_{1} \ldots q_{r} / T$ choices which are divisible by $T$ for each divisor $T$ of $q_{1} \ldots q_{r}$, we can form a weighted sum over all the divisors of $q_{1} \ldots q_{r}$ to determine precisely how many are not divisible by any such $T$. Specifically, the number of values of $k$ with $0 \leq k<q_{1} \ldots q_{r}$ such that $\left(1-k N, q_{1} \ldots q_{r}\right)=1$ is

$$
q_{1} q_{2} \ldots q_{r}+\sum_{n=1}^{r}\left[(-1)^{n} \sum_{r \geq i_{1}>i_{2}>\cdots>i_{n} \geq 1} \frac{q_{1} \ldots q_{r}}{q_{i_{1}} \ldots q_{i_{n}}}\right]=\left(q_{1}-1\right)\left(q_{2}-1\right) \ldots\left(q_{r}-1\right) .
$$

The result follows. 
In fact Lemma 3.6 can be generalized further so for $p=2$ we don't have $16 \mid n_{2}$ (though the proof is more technical). The next result is a generalization of the elementary abelian case and the proof is identical.

Lemma 3.7 If $n_{2}=p$ a prime, then there exists $K$ with $r / 2 \leq K \leq \min (\rho, \mathrm{r})$ such that any epimorphism $\eta: \Gamma \rightarrow G$ is equivalent to one and only one of those described below:

$$
\eta:= \begin{cases}\eta\left(\alpha_{i}\right)=\omega_{i} & i \leq K \\ \eta\left(\alpha_{i}\right)=0 & i>K \\ \eta\left(\beta_{i}\right)=\omega_{i+K} & i \leq r-K \\ \eta\left(\beta_{i}\right)=0 & i>r-K\end{cases}
$$

We now have enough information to complete the classification of fixed point free abelian actions up to genus 65 .

Example 3.8 First we eliminate all cases which can be classified by our results. Suppose $G$ has invariant factors $n_{1}, n_{2}, \ldots, n_{r}$. By Theorem 3.1, we may assume that $n_{1} \neq p$ for $p$ a prime and by Lemma 3.7, if the $p$-rank is greater than 1 , we may assume that $n_{2} \neq p$ for $p$ a prime either. Consequently, if $G$ has $p$-rank at least 3 and $n_{3}=a$, then $n_{2}=a b$ and $n_{1}=a b c$ where if $a$ is prime, then $b \neq 1$. Thus we would have $|G|=a^{3} b^{2} c \geq 64$ unless $a=2, b=2$ and $c=1$. However, in this case the invariant factors are 4, 4, 2 and we classified all such epimorphisms in Example 3.5. Thus we may assume $G$ has $p$-rank 2 .

For $p$-rank 2, Lemma 3.6 implies we may assume there exists a prime $p$ such that $p^{2} \mid n_{2}$ (and consequently $p^{2} \mid n_{1}$ ). If $p \geq 3$, then $|G| \geq 3^{4}$ and so the genus of the resulting surface will be at least 82 , so we may assume $p=2$. This means $n_{1}=4 k$ for some integer $k$. If $k>3$, the resulting surface will have genus greater than 65 , so we only need $n_{1}=n_{2}=4, n_{1}=8$ and $n_{2}=4$, and $n_{1}=12$ and $n_{2}=4$. However, in all these cases, there is a unique value $N$ for which $\left(N, n_{2}\right)>1$, so using the cohomology arguments of Lemma 3.6 and the general form given in Proposition 3.4, it is easy to check that there are just three classes:

$$
\begin{aligned}
& \eta_{1}:\left(\alpha_{1}, \beta_{1}, \alpha_{2}, \beta_{2}\right) \rightarrow\left(\omega_{1}, \omega_{2}, 0,0\right) \\
& \eta_{2}:\left(\alpha_{1}, \beta_{1}, \alpha_{2}, \beta_{2}\right) \rightarrow\left(\omega_{1}, 2 \omega_{2}, \omega_{2}, 0\right) \\
& \eta_{3}:\left(\alpha_{1}, \beta_{1}, \alpha_{2}, \beta_{2}\right) \rightarrow\left(\omega_{1}, 0, \omega_{2}, 0\right)
\end{aligned}
$$

\section{The totally ramified case}

Assume that $G=\mathbb{F}_{p}^{v}$ and that our action is purely ramified, namely $S / G$ has genus 0 , and that there are $r$ branch points. We are going to describe a method for computing 
the number of equivalence classes of actions of $G$. In each case there will be several small exceptional primes and a finite number of infinite families for which the number of actions is expressed as a polynomial in the prime. The infinite families are defined by the existence or nonexistence of certain roots of unity and so each family is typically the set of primes in an arithmetic progression. Unfortunately, our method depends on the enumeration of the finite subgroups of the symmetric group on $r$ points, so exact general results are impossible for all $p$-ranks and numbers of branch points. Thus, we will describe the general method, but only derive the precise details for 3 and 4 branch points (Table 10).

We may associate a generating set $\left\{C_{j} \mid 1 \leq j \leq r\right\}$ with a matrix $X$

$$
\left\{C_{j} \mid 1 \leq j \leq r\right\} \leftrightarrow\left[\begin{array}{llll}
X_{1} & X_{2} & \cdots & X_{r}
\end{array}\right]=X
$$

where $X$ satisfies the following conditions:

$X$ is a $v \times r$ matrix of rank $v$.

$$
X_{1}+X_{2}+\cdots+X_{r}=0 \text {. }
$$

Note that $1 \leq v<r$ and $r \geq 2$. Let us explicitly define the action as we shall need it in our calculations. Let $g \in G L\left(v, \mathbb{F}_{p}\right)$ be a $v \times v$ invertible matrix over $\mathbb{F}_{p}$, and $\alpha \in \Sigma_{r}$. Let $\pi_{\alpha}$ be the standard permutation matrix defined by

$$
\pi_{\alpha}=\left[\begin{array}{c}
E_{\alpha(1)} \\
E_{\alpha(2)} \\
\vdots \\
E_{\alpha(r)}
\end{array}\right]
$$

where $E_{1}, \ldots, E_{n}$ are the rows of the identity matrix. Then $\pi_{\alpha \beta}=\pi_{\alpha} \pi_{\beta}$ and the action $\operatorname{Aut}(G) \times \operatorname{Aut}(\Gamma)$ on the set of matrices $X$ is the action of $G L\left(v, \mathbb{F}_{p}\right) \times \Sigma_{r}$ given by

ie,

$$
\begin{gathered}
(g, \alpha) \cdot X=g X \pi_{\alpha}^{\top}, \\
g X \pi_{\alpha}^{\top}=\left[\begin{array}{llll}
g X_{\alpha(1)} & g X_{\alpha(2)} & \cdots & g X_{\alpha(r)}
\end{array}\right] .
\end{gathered}
$$

The problem of computing the number of equivalence classes is the same as computing the number of orbits under the given action. Before we start setting up the machinery for the computation of the orbits, let us prove a trivial case for all $p$-ranks.

Proposition 4.1 Suppose that the number of branch points is one more than the $p$-rank of $G$. Then there is a unique action of $G$. 
Proof Let $g=\left[\begin{array}{llll}X_{1} & X_{2} & \cdots & X_{v}\end{array}\right]$ be the square matrix obtained by dropping the last column of $X$. Since $X_{1}+X_{2}+\cdots+X_{r}=0$ then the column space of $g$ is the same as the column space of $X$. It follows the that $g$ is full rank and hence invertible. Then $g^{-1} X=\left[I_{v}-E_{v}\right]$ where $I_{r}$ is the $v \times v$ identity matrix and $E_{v}$ a column of ones.

\subsection{Orbit spaces via equisymmetry}

Let $\Omega$ be a finite set upon which the finite group $G$ (different from our $G$ above) acts. Consider the following standard constructs. Given a subset $Y \subseteq \Omega$ or $H \subseteq G$ let

$$
G_{Y}=\{g \in G \mid g x=x, \forall x \in Y\} \quad \text { and } \quad G_{x}=G_{\{x\}}
$$

and

$$
\Omega^{H}=\{x \in \Omega \mid g x=x, \forall g \in H\} \quad \text { and } \quad \Omega^{g}=\Omega^{\{g\}} .
$$

For $H \subseteq G$ let $\bar{H}$ denote the action closure of $H$, the largest group fixing all the points fixed by all elements of $H$,

$$
\bar{H}=G_{\Omega^{H}}=\bigcap_{x \in \Omega^{H}} G_{x} .
$$

Also $\bar{Y}$ denotes the action closure of a subset $Y \subseteq \Omega$, the set of all points fixed by all elements of $G$ fixing all of $Y$,

$$
\bar{Y}=\Omega^{G_{Y}}=\bigcap_{g \in G_{Y}} \Omega^{g} .
$$

Next for a subgroup $H \subseteq G$ let $\langle H\rangle$ denote the conjugacy class of subgroups of $G$ determined by $H$. The set of conjugacy classes has a partial order $\left\langle H_{1}\right\rangle \prec\left\langle H_{2}\right\rangle$ if and only if $H_{1} \subset g H_{2} g^{-1}$ for some $g \in G$. We make the following additional definitions.

Definition 4.2 Two points $x, y \in \Omega$ are called equi-isotropic if $G_{x}=G_{y}$ and equisymmetric if $G_{x}$ and $G_{y}$ are conjugate subgroups. The group $G_{x}$ is called the isotropy type of $x$ and $\left\langle G_{x}\right\rangle$ is called the symmetry type of $x$. If $H=G_{x}$ for some $x$ then the $H$-isotropic stratum or the isotropic stratum of $x$ is the set of all points with isotropy type $H$ :

$$
\stackrel{\circ}{\Omega}^{H}:=\left\{y \in \Omega \mid G_{y}=H\right\} .
$$

Likewise if $H=G_{x}$ for some $x$ then the $H$-equisymmetric stratum or the equisymmetric stratum of $x$ is the set of all points with symmetry type $\langle H\rangle$ :

$$
\left\langle\stackrel{\circ}{\Omega}^{H}\right\rangle:=\left\{y \in \Omega \mid G_{y} \in\langle H\rangle\right\} .
$$


The action closure $\Omega^{H}$ of $\AA^{H}$ is the set of points with $H$-isotropy or greater, ie, $\Omega^{H}=\left\{x \in \Omega \mid H \subseteq G_{x}\right\}$. It is easily seen that

$$
\Omega^{H}=\stackrel{\circ}{\Omega}^{H} \cup \bigcup_{K} \stackrel{\circ}{\Omega}^{K},
$$

a disjoint union for a set of closed groups $K$ strictly containing $K \supset H$. We can actually take the union over all subgroups $K \supset H$ fixing any point, though some of the $\stackrel{\circ}{\Omega}^{K}$ may be empty. We get a similar union

$$
\left\langle\Omega^{H}\right\rangle=\left\langle\stackrel{\circ}{\Omega}^{H}\right\rangle \cup \bigcup_{K}\left\langle\stackrel{\circ}{\Omega}^{K}\right\rangle
$$

for a set of closed point stabilizers $K$ such that $\langle K\rangle \succ\langle H\rangle$. The equations (16) and (17) may be rewritten to iteratively compute

$$
\begin{gathered}
\left|\stackrel{\Omega}{\Omega}^{H}\right|=\left|\Omega^{H}\right|-\sum_{K}\left|\stackrel{\circ}{\Omega}^{K}\right| \\
\left|\left\langle\stackrel{\circ}{\Omega}^{H}\right\rangle\right|=\left|\left\langle\Omega^{H}\right\rangle\right|-\sum_{K}\left|\left\langle\stackrel{\circ}{\Omega}^{K}\right\rangle\right|
\end{gathered}
$$

where the sums are over the same set of subgroups as above.

If $x$ and $y$ have the same symmetry type, ie, $G_{x}$ and $G_{y}$ are conjugate, then the orbits $G x$ and $G y$ have the same size $|G| /\left|G_{x}\right|$. Since $G_{g x}=g G_{x} g^{-1}$, then $g \stackrel{\circ}{\Omega}^{H}=\stackrel{\circ}{\Omega}^{g H g^{-1}}$ for a point stabilizer $H$ and so the set $\left\langle\AA^{H}\right\rangle$ is $G$-invariant. Indeed we have a disjoint union

$$
\left\langle\stackrel{\circ}{\Omega}^{H}\right\rangle=\bigcup_{K \in\langle H\rangle} \stackrel{\circ}{\Omega}^{K}=\bigcup_{g \in G / N_{G}(H)} g \stackrel{\circ}{\Omega}^{H}
$$

from which we get $\left|\left\langle\Omega^{H}\right\rangle\right|=|\langle H\rangle|\left|\Omega^{H}\right|$. Since $\left\langle\AA^{H}\right\rangle \rightarrow\left\langle\Omega^{H}\right\rangle / G$ is a $|G| /|H|$ to 1 map, we have

$$
\left|\left\langle\Omega^{H}\right\rangle / G\right|=\frac{|H|}{|G|}\left|\left\langle\AA^{H}\right\rangle\right|=\frac{|H|}{\left|N_{G}(H)\right|}\left|\stackrel{\circ}{\Omega}^{H}\right|
$$

and it follows that

$$
|\Omega / G|=\sum_{H}\left|\left\langle\stackrel{\circ}{\Omega}^{H}\right\rangle / G\right|=\sum_{H} \frac{|H|}{\left|N_{G}(H)\right|}\left|\stackrel{\circ}{\Omega}^{H}\right|,
$$

where $\langle H\rangle$ runs over all conjugacy classes of point stabilizers. So computing $|\Omega / G|$ is just a matter of determining the point stabilizers $H$ and the equisymmetric or the equi-isotropic strata. The cardinalities of the closed subsets $\Omega^{H}$ are generally easier to calculate directly which is why we use the above formulas. 


\subsection{Matrix formulation of Möbius inversion}

The iterative calculation of $|\Omega / G|$ via the formulas (18) and (20), which amounts to Möbius inversion, can be put into a very succinct matrix format. Consider the set of subgroups $H \subseteq G$ such that $\Omega^{H}$ is nonempty. For each conjugacy class of subgroups in this set select a representative and then list the subgroups $\langle\mathrm{id}\rangle=H_{1}, H_{2}, \ldots, H_{s}$, in such a way that $\left\langle H_{i}\right\rangle \subseteq\left\langle H_{j}\right\rangle \Longrightarrow i \leq j$. This can always be done, though generally not in a unique way. From Equation (16) we have:

$$
\begin{aligned}
\left|\Omega^{H_{i}}\right| & =\left|\stackrel{\circ}{\Omega}^{H_{i}}\right|+\sum_{K \supset H_{i}}\left|\stackrel{\circ}{\Omega}^{K}\right| \\
& =\left|\stackrel{\Omega}{\Omega}^{H_{i}}\right|+\sum_{j>i}\left(\sum_{K \in\left\langle H_{j}\right\rangle, H_{i} \subset K}\left|\stackrel{\circ}{\Omega}^{K}\right|\right)
\end{aligned}
$$

For $H, K \subseteq G$, let

$$
\begin{aligned}
& U(H, K)=\left\{g K g^{-1} \mid g \in G, H \subseteq g K g^{-1}\right\} \\
& D(H, K)=\left\{g^{-1} H g \mid g \in G, g^{-1} H g \subseteq K\right\}
\end{aligned}
$$

and set $u_{i, j}=\left|U\left(H_{i}, H_{j}\right)\right|, d_{i, j}=\left|D\left(H_{i}, H_{j}\right)\right|$. Observe that $u_{i, i}=d_{i, i}=1$ and $u_{i, j}=d_{i, j}=0$ if $i>j$ and from Lemma 4.3 below we have

$$
u_{i, j}=d_{i, j} \frac{\left|\left\langle H_{j}\right\rangle\right|}{\left|\left\langle H_{i}\right\rangle\right|} .
$$

Then Equation (21) can be rewritten as

$$
\begin{aligned}
\left|\Omega^{H_{i}}\right| & =\left|\AA^{H_{i}}\right|+\sum_{i<j} u_{i, j}\left|\stackrel{\circ}{\Omega}^{H_{j}}\right| \\
& =\left|\stackrel{\Omega}{\Omega}^{H_{i}}\right|+\sum_{i<j} d_{i, j} \frac{\left|\left\langle H_{j}\right\rangle\right|}{\left|\left\langle H_{i}\right\rangle\right|}\left|\stackrel{\Omega}{\Omega}^{H_{j}}\right|
\end{aligned}
$$

and we have the equality

$$
\left|\left\langle\AA^{H_{i}}\right\rangle\right|=\left|\stackrel{\circ}{\Omega}^{H_{i}}\right|\left|\left\langle H_{i}\right\rangle\right| \text {. }
$$

Now let us convert to matrix notation. Define

$$
\begin{aligned}
U & =\left[u_{i, j}\right], \quad D=\left[d_{i, j}\right] \\
S & =\operatorname{diag}\left(\left|\left\langle H_{1}\right\rangle\right|,\left|\left\langle H_{2}\right\rangle\right|, \ldots,\left|\left\langle H_{S}\right\rangle\right|\right) \\
N & =\operatorname{diag}\left(\left|N_{G}\left(H_{1}\right)\right|,\left|N_{G}\left(H_{2}\right)\right|, \ldots,\left|N_{G}\left(H_{2}\right)\right|\right) \\
T & =\operatorname{diag}\left(\left|H_{1}\right|,\left|H_{2}\right|, \ldots,\left|H_{S}\right|\right) .
\end{aligned}
$$


The matrices $U=\left[u_{i, j}\right]$ and $D=\left[d_{i, j}\right]$ are upper triangular with 1 's on the diagonal. It follows from (24) that $U=S^{-1} D S$. Let $L, L^{\circ}, E^{\circ}, O^{\circ}$ be the vectors

$$
\begin{aligned}
& L=\left[\begin{array}{llll}
\left|\Omega^{H_{1}}\right| & \left|\Omega^{H_{2}}\right| & \cdots & \left|\Omega^{H_{s}}\right|
\end{array}\right]^{\top} \\
& L^{\circ}=\left[\left|\stackrel{\Omega}{\Omega}^{H_{1}}\right|\left|\stackrel{\Omega}{\Omega}^{H_{2}}\right| \cdots\left|\stackrel{\circ}{\Omega}^{H_{s}}\right|\right]^{\top} \\
& E^{\circ}=\left[\left|\left\langle\stackrel{\circ}{\Omega}^{H_{1}}\right\rangle\right|\left|\left\langle\stackrel{\circ}{\Omega}^{H_{2}}\right\rangle\right| \cdots\left|{\stackrel{\circ}{\Omega} H_{s}}\right\rangle\right]^{\top} \\
& O^{\circ}=\left[\left|\left\langle\Omega^{H_{1}}\right\rangle / G\right|\left|\left\langle\Omega^{H_{2}}\right\rangle / G\right| \cdots\left|\left\langle\stackrel{\Omega}{ }^{H_{s}}\right\rangle / G\right|\right]^{\top} .
\end{aligned}
$$

The vectors $E^{\circ}$ and $O^{\circ}$ are the cardinalities of the various equisymmetric strata and corresponding orbits, $|\Omega / G|$ is simply the sum of the entries of $O^{\circ}$. The equations (21) to (26) can be translated into the following matrix equations:

$$
\begin{aligned}
L & =U L^{\circ}=S^{-1} D S L^{\circ} \\
L^{\circ} & =U^{-1} L=S^{-1} D^{-1} S L \\
E^{\circ} & =S L^{\circ}=S S^{-1} D^{-1} S L=D^{-1} S L \\
O^{\circ} & =\frac{1}{|G|} T E^{\circ}=\frac{1}{|G|} T D^{-1} S L .
\end{aligned}
$$

Finally we have the matrix equation $N^{-1}=\frac{1}{|G|} S$ relating normalizers and conjugacy classes resulting in our final equation

$$
O^{\circ}=T D^{-1} N^{-1} L
$$

The following lemma relates the $u_{i, j}$ and $d_{i, j}$.

Lemma 4.3 Let $H \subseteq K$ be subgroups of a finite group $G$. Let $U(H, K)$ and $D(H, K)$ be the sets defined in equations (22) and (23). Then

$$
U(H, K)|\langle H\rangle|=|D(H, K)||\langle K\rangle| .
$$

Proof Let $P=\{(S, T) \in\langle H\rangle \times\langle K\rangle \mid S \subseteq T\}$. By considering the projections $P \rightarrow\langle H\rangle, P \rightarrow\langle K\rangle$ we see that $|U(H, K)||\langle H\rangle|=|P|=|D(H, K)||\langle K\rangle|$.

Remark 4.4 The entries of $D$ can be found by enumerating the subgroups of $H_{j}$ and then determining to which $H_{i}$ they are conjugate. In our calculations we will just work with subgroups of small symmetric groups. 


\subsection{Structure of stabilizers}

Let $\Omega$ be the set of matrices $X$ satisfying conditions (13) and (14), and $G=G L\left(v, \mathbb{F}_{p}\right) \times$ $\Sigma_{r}$ with the action given in Equation (15). There are two canonical representations $\rho_{1}: G \rightarrow G L\left(v, \mathbb{F}_{p}\right),(g, \alpha) \mapsto g$ and $\rho_{2}: G \rightarrow \Sigma_{r} \rightarrow G L\left(r, \mathbb{F}_{p}\right),(g, \alpha) \mapsto \pi_{\alpha}$. We are going to describe the structure of stabilizers $H \subseteq G$ in terms of the two representations. Let $X_{0} \in \Omega$ and suppose that $H$ is a subgroup fixing $X_{0}$, (the subgroup need not be action closed). If $X$ is any matrix stabilized by $H$ then $\rho_{1}(h) X \rho_{2}^{\top}(h)=X, h \in H$ or

$$
\rho_{1}(h) X=X \rho_{2}(h), h \in H,
$$

ie, $X \in \operatorname{Hom}_{H}\left(\mathbb{F}_{p}^{r}, \mathbb{F}_{p}^{v}\right)$ is an intertwining operator. Now, the restricted map $p_{2}: H \rightarrow$ $\Sigma_{r}$, is one to one, for if $(g, \alpha) \in \operatorname{ker}\left(p_{2}\right)$ then $\alpha=$ id and $X_{0}=g X_{0}$. Since $X_{0}$ has rank $v$ then $g=I$. Let $H^{\prime}=p_{2}(H)$ denote the isomorphic image of $H$ in $\Sigma_{r}$. Reversing the process, we can construct candidate stabilizers by selecting a subgroup $H^{\prime} \subseteq \Sigma_{r}$, and a suitable representation $q=\rho_{1} \circ p_{2}^{-1}: H^{\prime} \rightarrow G L\left(v, \mathbb{F}_{p}\right)$ and then construct $H$ by

$$
H=\left\{(q(\alpha), \alpha) \mid \alpha \in H^{\prime}\right\}
$$

It is not clear that an arbitrary $H$ so constructed fixes any matrices in $\Omega$, in fact the representation $q$ must satisfy certain properties which we proceed to discuss.

For the remainder of our discussion we are going to assume that $p \nmid|H|$, so that we may use the theory of reducible representations to aid our computations. Since $|H|$ divides $r$ ! we are only excluding the small primes $p \leq r$. Both $H$-modules $\mathbb{F}_{p}^{v}$, $\mathbb{F}_{p}^{r}$ can be rewritten as a direct sum of $\mathbb{F}_{p}$-irreducibles, $\mathbb{F}_{p}^{v}=V_{1} \oplus V_{2} \oplus \cdots \oplus V_{m}$, $\mathbb{F}_{p}^{r}=W_{1} \oplus W_{2} \oplus \cdots \oplus W_{n}$. By a change in coordinates, we may write

$$
\begin{aligned}
A^{-1} \rho_{1}(h) A & =\left[\begin{array}{cccc}
\phi_{1}(h) & 0 & \cdots & 0 \\
0 & \phi_{2}(h) & \cdots & 0 \\
\vdots & \vdots & \ddots & \vdots \\
0 & 0 & \cdots & \phi_{m}(h)
\end{array}\right] \\
B^{-1} \rho_{2}(h) B & =\left[\begin{array}{cccc}
\psi_{1}(h) & 0 & \cdots & 0 \\
0 & \psi_{2}(h) & \cdots & 0 \\
\vdots & \vdots & \ddots & \vdots \\
0 & 0 & \cdots & \psi_{n}(h)
\end{array}\right]
\end{aligned}
$$

Algebraic 83 Geometric Topology, Volume 7 (2007) 
where each $\phi_{i}$, and $\psi_{j}$ is irreducible on $V_{i}$ and $W_{j}$ respectively. Writing $Y=A^{-1} X B$ in corresponding block form

$$
Y=A^{-1} X B=\left[\begin{array}{cccc}
Y_{1,1} & Y_{1,2} & \cdots & Y_{1, n} \\
Y_{2,2} & Y_{2,2} & \cdots & Y_{2, n} \\
\vdots & \vdots & \ddots & \vdots \\
Y_{m, 1} & Y_{m, 2} & \cdots & Y_{m, n}
\end{array}\right]
$$

the intertwining property has an equivalent formulation

$$
\rho_{1}(h) X=X \rho_{2}(h) \Longleftrightarrow \forall_{i, j} \phi_{i}(h) Y_{i, j}=Y_{i, j} \psi_{j}(h),
$$

and hence we may consider $Y_{i, j} \in \operatorname{Hom}_{H}\left(W_{j}, V_{i}\right)$. Now, as $H$ acts irreducibly on $V_{i}$ and $W_{j}$, then $Y_{i, j}=0$ if $\phi_{i}, \psi_{j}$ are inequivalent. If $\phi_{i}$ and $\psi_{j}$ are equivalent then $Y_{i, j}$ is invertible, if nonzero, by Schur's Lemma. The number of invertible $Y_{i . j}$ is $p^{k}-1$, where $k$ is the number of irreducibles into which $\phi_{i}=\psi_{j}$ splits in the algebraic closure of $\mathbb{F}_{p}$. We choose $\psi_{n}$ to be the trivial representation acting on the space $W_{n}=\left\langle\left[\begin{array}{llll}1 & 1 & \ldots & 1\end{array}\right]^{\top}\right\rangle$. Since $X\left[\begin{array}{llll}1 & 1 & \ldots & 1\end{array}\right]^{\top}=0$ then $Y_{i, n}=0$ for all $i$. For convenience we introduce a special name for the representation on $W_{1} \oplus \cdots \oplus W_{n-1}$.

Definition 4.5 For a subgroup $H^{\prime} \subseteq \Sigma_{r}$ acting naturally on the space $\mathbb{F}_{p}^{r}$ write $\mathbb{F}_{p}^{r}=W \oplus\left\langle\left[\begin{array}{llll}1 & 1 & \ldots & 1\end{array}\right]^{\top}\right\rangle$ as a direct sum of subrepresentations. If $\pi$ denotes the natural representation of $H^{\prime}$, we call $\pi_{W}$, the natural representation restricted to $W$, the reduced natural representation of $H^{\prime}$ and denote it by $\bar{\pi}$. If $p \nmid r$ then may take $W=\left\{\left(x_{1}, \ldots, x_{r}\right) \in \mathbb{F}_{p}^{r} \mid x_{1}+\cdots+x_{r}=0\right\}$.

Remark 4.6 Though a bit pedantic, we remind ourselves on how to construct $A$ and $B$ since the details will be used in a later proof. The matrix $A$ may be constructed by choosing column vectors that span $V_{1}$ then adding vectors that span $V_{2}$ and so on to get $A=\left[\begin{array}{llll}A_{1} & A_{2} & \cdots & A_{m}\end{array}\right]$, where $A_{i}$ is a $v \times v_{i}$ matrix. Construct $B=$ $\left[\begin{array}{llll}B_{1} & B_{2} & \cdots & B_{n}\end{array}\right]$, similarly. Now write $A^{-1}=\left[\begin{array}{ll}\left(A_{1}^{*}\right)^{\top} & \left(A_{2}^{*}\right)^{\top} \cdots\left(A_{m}^{*}\right)^{\top}\end{array}\right]^{\top}$ as a column of matrices with $A_{i}^{*}$ of size $v_{i} \times v$. Define the $B_{j}^{*}$ similarly. We have the following transformation formula:

$$
Y_{i, j}=A_{i}^{*} X B_{j}, \quad X=\sum_{i, j} A_{i} Y_{i, j} B_{j}^{*}
$$

The next definition helps us characterize the representations we are looking for in the defining Equation (30).

Definition 4.7 Let $H$ be a finite group and $k$ a field whose characteristic is coprime to $|H|$. Let $\rho_{1}, \rho_{2}$ be two representations of finite degree on $k$ vector spaces $V, W$. We 
say that $\rho_{2}$ dominates $\rho_{1}\left(\rho_{1} \preceq \rho_{2}\right)$ if and only if for each $k$-irreducible representation $\phi$ of $H,\left\langle\phi, \rho_{1}\right\rangle \leq\left\langle\phi, \rho_{2}\right\rangle$ where $\langle\phi, \rho\rangle$ is the number of times the representation $\phi$ is contained in a representation $\rho$ of $H$.

Proposition 4.8 Let $H, k$ and $\rho_{1}, \rho_{2}$ be as in Definition 4.7. Then $\rho_{1} \preceq \rho_{2}$ if and only if the space of intertwining operators $\operatorname{Hom}_{H}(W, V)$ contains a surjective map. Similarly $\rho_{2} \preceq \rho_{1}$ if and only if $\operatorname{Hom}_{H}(W, V)$ contains an injective map.

When restated in terms $H$-modules over $\mathbb{F}_{p}$ the proposition is self-evident. In proof of Proposition 4.10, which characterizes the representations defining subgroups that fix a point, we will see that the dominance condition $\rho_{1} \preceq \rho_{2}$ is equivalent to the existence of an intertwining operator $X$ with linearly independent rows.

Remark 4.9 Suppose the action of $H$ has several orbits on $\{1,2, \ldots, r\}$ and that by selecting a suitable conjugate of $H^{\prime}$ each orbit is an interval of $t$ integers

$$
\{s, s+1 \ldots, s+t-1\} .
$$

We may write

$$
X=\left[\begin{array}{llll}
Z_{1} & Z_{2} & \cdots & Z_{l}
\end{array}\right]
$$

as a block matrix where each block is defined by an orbit and hence invariant under $H^{\prime}$. Then $\rho_{1}$ must satisfy

$$
\rho_{1}(h) Z_{k}=Z_{k} \rho_{2, k}(h), k=1, \ldots, l
$$

where $\rho_{2, k}(h)$ is the induced permutation matrix on the orbit defining $Z_{k}$. We use these representations to characterize when a pair $H^{\prime} \subseteq \Sigma_{r}, q: H^{\prime} \rightarrow G L\left(v, \mathbb{F}_{p}\right)$ fixes a point in $\Omega$.

Proposition 4.10 Assume that $v>1$ if $p=2$. Let $H^{\prime} \subseteq \Sigma_{r}$ such that $p \nmid|H|$, let $q: H^{\prime} \rightarrow G L\left(v, \mathbb{F}_{p}\right)$ be an arbitrary representation, and let $H=\left\{(q(\alpha), \alpha) \mid \alpha \in H^{\prime}\right\}$. Let $\rho, \bar{\rho}$ be the standard and reduced representations of $H^{\prime}$ afforded by $H^{\prime} \subseteq \Sigma_{r}$, and $\rho_{i}=\rho_{2, i} \circ p_{2}^{-1}$ be the representations of $H^{\prime}$ determined by the orbits of $H^{\prime}$ on $\{1,2, \ldots, r\}$. Then $H$ fixes a point in $\Omega$ if and only if all the following conditions hold:

- $q$ is dominated by $\bar{\rho}$.

- $q$ and $\rho_{k}$ have a common irreducible for $k=1, \ldots, l$. 
Proof By taking a conjugate of $H^{\prime}$ we may assume that the orbits occur in intervals as Remark 4.9. Let $\rho_{1}$ and $\rho_{2}, \rho_{2, k}, Z_{k}$ be as defined previously so that $\rho_{1}=q \circ p_{2}$, and, $\rho_{2}=\rho \circ p_{2}, \overline{\rho_{2}}=\bar{\rho} \circ p_{2}, \rho_{2, i}=\rho_{i} \circ p_{2}$. To show that $H$ fixes an element of $\Omega$ we need to find a $v \times r$ matrix $X$ such that

- $\rho_{1}(h) X=X \rho_{2}(h), h \in H$,

- $X_{1}+X_{2}+\cdots+X_{r}=0$,

- $X$ is surjective, ie, has linearly independent rows,

- each column $X_{i}$ is nonzero.

We leave it to the reader to show that the conditions are necessary, we show how to construct an $X$ if the conditions hold. Our proof depends on carefully setting up the diagonalizing matrices $A$ and $B$, and then carefully selecting the $Y_{i, j}$. Because $(g, \alpha)$ in $H$ acts on a column $X_{j}$ by $X_{j} \rightarrow g X_{\alpha(j)}$, then a $Z_{k}$ defined by an $H^{\prime}$ orbit will be nonzero if and only if all the columns of the given $Z_{k}$ are nonzero. Thus we merely need to construct $X$ satisfying the first three bullets and all $Z_{i}$ nonzero. The diagonalizing matrix $A$ may be constructed according to any decomposition, but we need to be more careful with $B$. Decompose $\rho_{2,1}$ into $H$-irreducibles and then place the corresponding basis vectors into $B$ as columns as described in Remark 4.9. Next decompose $\rho_{2,2}$ and add the basis vectors as columns, and continue on to the last orbit. Each orbit determines a unique trivial subrepresentation of the $\rho_{2, k}$. A spanning vector for this subrepresentation is the vector with 1's in the locations corresponding to the orbit and zeros elsewhere. By construction, for each of these vectors, $B$ contains a column which is a scalar multiple of this vector. We assume that the scalar is 1 and that the last column of $B$ is the vector corresponding to the orbit defining $\rho_{2, l}$. Now the sum of these columns is $\left[\begin{array}{llll}1 & 1 & \ldots & 1\end{array}\right]^{\top}$. If we replace the last column of $B$ with the vector $\left[\begin{array}{llll}1 & 1 & \ldots & 1\end{array}\right]^{\top}$ we obtain a matrix $C$ with the same column span as $B$ and hence $C$ is invertible. Moreover as we exchanged one invariant vector for another $B^{-1} \rho_{2}(h) B=C^{-1} \rho_{2}(h) C$. Thus we can assume that $B$ has $B_{n}=\left[\begin{array}{llll}1 & 1 & \ldots & 1\end{array}\right]^{\top}$ as its last column.

Now we are going to further modify $A$ and $B$ as follows. We assume that we have selected our representations $\phi_{i}$ and $\psi_{j}$ so that if any two are equivalent then they are equal. This can be achieved by modifying the columns of $A$ or $B$ corresponding to the $H$-invariant subspace corresponding to a $\phi_{i}$ or $\psi_{j}$. Note that we do not need to alter the last column of $B$. In this circumstance we define the matrix components of $Y$ in Equation (31):

$$
\begin{aligned}
& Y_{i, j}=y_{i, j} I_{v_{i}} \text { if } \phi_{i}=\psi_{j} \\
& Y_{i, j}=0, \text { otherwise. }
\end{aligned}
$$


By Equation (32) the above equations always define an intertwining operator constructed via Equation (33). Now $X_{1}+X_{2}+\cdots+X_{r}=X B_{n}$, where $B_{n}=\left[\begin{array}{llll}1 & 1 & \ldots & 1\end{array}\right]^{\top}$. But $X B_{n}$ equals the last column of $X B=A Y$. It follows then that $X_{1}+X_{2}+\cdots+X_{r}=0$ if and only if the last column of $Y$ is zero, ie, $Y_{i . n}=0$ for all $i$. Next we modify $Y$ so that $X$ is surjective. From the hypotheses $\overline{\rho_{2}}$ dominates $\rho_{1}$, and hence for each irreducible $\phi_{i}$ there can be chosen a $\psi_{j(i)}$ with $\phi_{i}=\psi_{j(i)}, j(i)<n$, and such that for distinct $i_{1}, i_{2}$ we have $j\left(i_{i}\right) \neq j\left(i_{i}\right)$. If we select $y_{i, j(i)} \neq 0$ and all other $y_{i, j}=0$ then the resulting matrix $Y$, and consequently $X$, has linearly independent rows. (This argument shows surjectivity in Proposition 4.8.)

Finally, we show that the $Y_{i, j}$ can be chosen so that each $Z_{k}$ in Equation (34) is nonzero. By Equation (34) $Y=A^{-1} X B=\left[\begin{array}{lllll}A^{-1} Z_{1} B & A^{-1} Z_{2} B & \cdots & A^{-1} Z_{l} B\end{array}\right]$ so if some $Z_{i}=0$ then $Y$ has a block column equal to zero and by construction there is a set $J_{0}$ of $j$ 's such that $Y_{i, j}=0,1 \leq i \leq m, j \in J_{0}$. The $J_{0}$ consists of all $j$ such that $\psi_{j}$ comes from the subspace determined by the orbit corresponding to $Z_{k}$. By assumption $\rho_{1}$ and $\rho_{2, k}$ have a common irreducible. Then we can set some $Y_{i, j}=y_{i, j} I_{v_{i}}$ for $i$ and a $j \in J_{0}$, and any $y_{i, j} \neq 0$. The modified $Y$ will still define an intertwining operator, and $Y$ will remain full rank since we are modifying columns that were initially zero. Thus we can guarantee that all $Z_{k}$ are nonzero, and still leave $X$ a surjective intertwining operator. The potential problem is if we were forced to change something in the last column of $Y$. This can only happen if the trivial representation is the only common irreducible between $\rho_{2, l}$ and $\overline{\rho_{2}}$. Now suppose that there is another $\rho_{2, l^{\prime}}$ such that $\overline{\rho_{2}}$ and $\rho_{2, l^{\prime}}$ have a common nontrivial irreducible. Then by taking a conjugate of $H^{\prime}$ we can switch $\rho_{2, l}$ and $\rho_{2, l^{\prime}}$ so that the $Y_{i, j}$ can be adjusted without affecting the last column of $Y$. Now suppose that even switching in not possible. Then it follows that $q$ is trivial and that the number of orbits is greater than $v$. Define $X$ as follows:

$$
X=\left[\begin{array}{ccccccc}
E_{r_{1}} & 0 & \cdots & 0 & r_{1} y_{1} E_{r_{v+1}} & \cdots & r_{1} y_{l-v} E_{r_{l}} \\
0 & E_{r_{2}} & \cdots & 0 & r_{2} y_{1} E_{r_{v+1}} & \cdots & r_{2} y_{l-v} E_{r_{l}} \\
\vdots & \vdots & \ddots & 0 & \vdots & \ddots & \vdots \\
0 & 0 & 0 & E_{r_{v}} & r_{v} y_{1} E_{r_{v+1}} & \cdots & r_{v} y_{l-v} E_{r_{l}}
\end{array}\right]
$$

where $E_{n}$ is a row matrix of 1's, $r_{s}$ is the size of the $s$-th orbit and

$$
y_{1} r_{v+1}+\cdots+y_{l-v} r_{l}=-1, y_{i} \neq 0, r_{i} \neq 0 .
$$

Assuming that the $y_{j}$ 's satisfy the given constraints and at least one of the $r_{i}$ 's is nonzero then $X$ meets all the requirements. Since $p$ is coprime to $|H|$, none of the $r_{i}$ 's are zero. Set $s=l-v>0$ and assume that $p \neq 2$. According to Proposition 4.16 in the next section, only $\left((p-1)^{S}-(-1)^{S}\right) / p+(-1)^{S}$ of the $(p-1)^{s}$ selections of $y_{i}$ sum to zero. Thus at least one of the sums in Equation (37) is nonzero and it may 
be scaled to equal -1 . If $p=2$ and $v>1$ then choose $X$ of the following form:

$$
X=\left[\begin{array}{cccccccc}
E_{r_{1}} & y E_{r_{1}} & \cdots & 0 & E_{r_{v+1}} & E_{r_{v+2}} & \cdots & E_{r_{l}} \\
0 & E_{r_{1}} & \cdots & 0 & E_{r_{v+1}} & 0 & \cdots & 0 \\
\vdots & \vdots & \ddots & 0 & \vdots & \vdots & \ddots & \vdots \\
0 & 0 & 0 & E_{r_{v}} & E_{r_{v+1}} & 0 & \cdots & 0
\end{array}\right]
$$

Since all the $r_{i}$ are odd then $y$ may be chosen so that all row sums are zero and that no column is zero.

\subsection{Normalizers and conjugates}

Let us describe how to compute normalizers of subgroups $G L\left(v, \mathbb{F}_{p}\right) \times \Sigma_{r}$ fixing a point. Note that we are considering the action of $G L\left(v, \mathbb{F}_{p}\right) \times \Sigma_{r}$ on generating vectors, not the related action $\operatorname{Aut}(G) \times \operatorname{Aut}(\Gamma)$ on Teichmüller space. Suppose that $(g, \alpha)$ normalizes $H$. Then for $(q(\beta), \beta) \in H,(g, \alpha)(q(\beta), \beta)\left(g^{-1}, \alpha^{-1}\right)=\left(g q(\beta) g^{-1}, \alpha \beta \alpha^{-1}\right)=$ $\left(q\left(\alpha \beta \alpha^{-1}\right), \alpha \beta \alpha^{-1}\right) \in H$ so we must have

$$
\begin{gathered}
\alpha \beta \alpha^{-1} \in H^{\prime} \\
g q(\beta) g^{-1}=q\left(\alpha \beta \alpha^{-1}\right) .
\end{gathered}
$$

Thus we must already have $\alpha \in N_{\Sigma_{r}}\left(H^{\prime}\right)=N^{\prime}$. The normalizer $N^{\prime}$ permutes the representations of $H^{\prime}$ by the formula

$$
q^{\alpha}(\beta)=q\left(\alpha \beta \alpha^{-1}\right)
$$

so there is $(g, \alpha)$ normalizing $H$ if and only if $q$ and $q^{\alpha}$ are equivalent over $\mathbb{F}_{p}$. In terms of characters, $\alpha$ must fix the character of $q$. Let $N^{\prime \prime}$ be the subgroup satisfying $H^{\prime} \subseteq N^{\prime \prime} \subseteq N^{\prime}$ and fixing the character of $q$. If $\left(g_{1}, \alpha\right),\left(g_{2}, \alpha\right)$ both belong to the normalizer of $H$ then Equation (38) implies $g_{1} q(\beta) g_{1}^{-1}=q\left(\alpha \beta \alpha^{-1}\right)=$ $g_{2} q(\beta) g^{-1}$ or $q(\beta)=\left(g_{1}^{-1} g_{2}\right) q(\beta)\left(g_{1}^{-1} g_{2}\right)^{-1}$ for all $\beta \in H^{\prime}$. Thus, $h=g_{1}^{-1} g_{2} \in$ $Z=Z_{G L\left(v, \mathbb{F}_{p}\right)}\left(q\left(H^{\prime}\right)\right)$. It follows that the normalizer of $H$ contains $Z$ as a subgroup with quotient $N^{\prime \prime}$ and so the normalizer has size $|Z|\left|N^{\prime \prime}\right|$.

Two subgroups $H_{1}$ and $H_{2}$ of $G L\left(v, \mathbb{F}_{p}\right) \times \Sigma_{r}$ fixing a point are conjugate if and only $H_{2}^{\prime}=\alpha H_{2}^{\prime} a^{-1}$ and the two representations $q^{\prime}$ and $q$ satisfy

$$
q^{\prime}\left(\alpha \beta \alpha^{-1}\right)=g q(\beta) g^{-1}
$$

for some $g \in G L\left(v, \mathbb{F}_{p}\right)$. 


\subsection{Fixed point subsets and strata}

There are three ways we shall consider to calculate the size of fixed point subsets. We illustrate the first two by example with $v=2, r=4, H^{\prime}=\langle(1,2)(3,4)\rangle$ and $q((1,2)(3,4))=\left[\begin{array}{cc}1 & 0 \\ 0 & -1\end{array}\right]$. The first method is by brute force and can easily be implemented by computer.

Example 4.11 A typical $X=\left[\begin{array}{llll}x_{1} & x_{2} & x_{3} & x_{4} \\ y_{1} & y_{2} & y_{3} & y_{4}\end{array}\right]$ must satisfy the equations

$$
\begin{aligned}
& x_{1}+x_{2}+x_{3}+x_{4}=y_{1}+y_{2}+y_{3}+y_{4}=0 \\
& x_{1}-x_{2}=x_{3}+x_{4}=y_{1}-y_{2}=y_{3}+y_{4}=0
\end{aligned}
$$

and so $X=\left[\begin{array}{cccc}x & x & -x & -x \\ y & -y & z & -z\end{array}\right]$. Clearly $x \neq 0$ and at least one of the six $2 \times 2$ minors must be nonzero, ie, one of $x z, x(y-z), x(y+z), x y$ must be nonzero. It follows that our conditions are $x \neq 0$ and $(y, z) \neq(0,0)$ and the number of valid $X$ 's is $(p-1)\left(p^{2}-1\right)$.

A drawback to this method is that the representation must be constructed. However, we note that it works if $p$ divides the order of a stabilizer. The second method uses the "diagonalized" form of the intertwining operator $Y=A^{-1} X B$, and can usually be determined by inspection of the characters of $q$ and $\bar{\rho}$.

Example 4.12 Observe that the character of $q$ is $\chi_{0}+\chi_{1}$ and $\bar{\rho}=\chi_{0}+2 \chi_{1}$, where $\chi_{0}$ and $\chi_{1}$ are the trivial and nontrivial characters of $H^{\prime}$ respectively. The matrices $A, B$, and $Y$ can be chosen as:

$$
A=\left[\begin{array}{ll}
1 & 0 \\
0 & 1
\end{array}\right], \quad B=\left[\begin{array}{cccc}
1 & 1 & 0 & 1 \\
1 & -1 & 0 & 1 \\
0 & 0 & 1 & 1 \\
0 & 0 & -1 & 1
\end{array}\right], Y=\left[\begin{array}{llll}
x & 0 & 0 & 0 \\
0 & y & z & 0
\end{array}\right] .
$$

We need $x \neq 0$ and $(y, z) \neq(0,0)$ giving the same result as Example 4.11. Note that we don't need to know $A$ and $B$ explicitly to determine $Y$, just the characters.

The two previous methods are useful when the fixed point set has small dimension and the nonvanishing polynomials are simple. At the other extreme we need to calculate the fixed point set for the trivial point stabilizer. As a starting point we need to calculate the size of $\Omega$. We can calculate the number of vectors by inclusion-exclusion followed by a specialized Möbius inversion. Let $V$ be an arbitrary vector space of dimension $v$ over $\mathbb{F}_{p}$ and let

$$
\begin{aligned}
& \bar{\Omega}^{r}(V)=\left\{X=\left(X_{1}, \ldots, X_{r}\right) \in V^{r} \mid X_{i} \neq 0, i=1, \ldots, r, X_{1}+X_{2}+\cdots+X_{r}=0\right\} \\
& \Omega^{r}(V)=\left\{X \in \bar{\Omega}^{r}(V) \mid V=\left\langle X_{1}, \ldots, X_{r}\right\rangle\right\}
\end{aligned}
$$


and set $\bar{\Omega}^{v, r}=\bar{\Omega}^{r}\left(\mathbb{F}_{p}^{v}\right), \Omega^{v, r}=\Omega^{r}\left(\mathbb{F}_{p}^{v}\right)$. Clearly the cardinalities of the sets depend only on $r$ and $v=\operatorname{dim} V$.

Proposition 4.13 Let $\bar{\Omega}^{r}(V)$ and $\Omega^{r}(V)$ be defined as above and set

$$
\bar{\omega}(v, r)=\left|\bar{\Omega}^{r}(V)\right|, \quad \omega(v, r)=\left|\Omega^{r}(V)\right| .
$$

Then we have

$$
\begin{aligned}
\bar{\omega}(v, r) & =\frac{\left(p^{v}-1\right)^{r}-(-1)^{r}}{p^{v}}+(-1)^{r} \\
\omega(1, r) & =\bar{\omega}(1, r) \\
\omega(v, r) & =\bar{\omega}(v, r)-\sum_{l=1}^{v-1} n_{v, l} \omega(l, r) \\
n_{v, l} & =\left(\prod_{j=l+1}^{v}\left(p^{j}-1\right)\right) /\left(\prod_{j=1}^{v-l}\left(p^{j}-1\right)\right)
\end{aligned}
$$

where

is the number of subspaces $L$ of dimension $l$ in $V$.

Proof Let

$$
\begin{aligned}
\bar{\Omega}_{0} & =\left\{\left(X_{1}, \ldots, X_{r}\right) \in V^{r} \mid X_{1}+X_{2}+\cdots+X_{r}=0\right\} \\
\bar{\Omega}_{i} & =\left\{\left(X_{1}, \ldots, X_{r}\right) \in V^{r} \mid X_{i}=0, X_{1}+X_{2}+\cdots+X_{r}=0\right\}, i=1, \ldots, r \\
\bar{\Omega} & =\left\{\left(X_{1}, \ldots, X_{r}\right) \in V^{r} \mid X_{i} \neq 0, i=1, \ldots, r, X_{1}+X_{2}+\cdots+X_{r}=0\right\}
\end{aligned}
$$

so that $\bar{\Omega}^{r}(V)=\bar{\Omega}=\bar{\Omega}_{0}-\bigcup_{i} \bar{\Omega}_{i}$. Then by inclusion-exclusion:

$$
\begin{aligned}
\bar{\omega}(v, r)= & |\bar{\Omega}| \\
= & \left|\bar{\Omega}_{0}\right|-\sum_{i}\left|\bar{\Omega}_{i}\right|+\sum_{i<j}\left|\bar{\Omega}_{i} \cap \bar{\Omega}_{j}\right|-\sum_{i<j<k}\left|\bar{\Omega}_{i} \cap \bar{\Omega}_{j} \cap \bar{\Omega}_{k}\right|+\cdots \\
& \quad+(-1)^{r}\left|\bar{\Omega}_{i} \cap \cdots \cap \bar{\Omega}_{r}\right| \\
= & p^{v(r-1)}-\left(\begin{array}{c}
r \\
1
\end{array}\right) p^{v(r-2)}+\left(\begin{array}{l}
r \\
2
\end{array}\right) p^{v(r-3)}-\left(\begin{array}{c}
r \\
3
\end{array}\right) p^{v(r-3)}+\cdots \\
& \quad+(-1)^{r-1}\left(\begin{array}{c}
r \\
r-1
\end{array}\right)+(-1)^{r} \\
= & \frac{\left(p^{v}-1\right)^{r}-(-1)^{r}}{p^{v}}+(-1)^{r}
\end{aligned}
$$

Algebraic $\mathcal{E} \mathcal{G}$ Geometric Topology, Volume 7 (2007) 
Next let $L$ be denote an arbitrary proper subspace of $V$ of dimension $l$. Then

$$
\bar{\Omega}^{r}(V)=\Omega^{r}(V) \cup \bigcup_{0 \subset L \subset V} \Omega^{r}(L) .
$$

It follows that

$$
\begin{aligned}
\bar{\omega}(v, r) & =\left|\bar{\Omega}^{r}(V)\right|=\left|\Omega^{r}(V)\right|+\sum_{l=1}^{v-1} \sum_{\operatorname{dim} L=l} \Omega^{r}(L) \\
& =\left|\Omega^{r}\left(\mathbb{F}_{p}^{v}\right)\right|+\sum_{l=1}^{v-1} n_{v, l}\left|\Omega^{r}\left(\mathbb{F}_{p}^{l}\right)\right| \\
& =\omega(v, r)+\sum_{l=1}^{v-1} n_{v, l} \omega(l, r) .
\end{aligned}
$$

The remaining formulas in equations (39) follow immediately. Finally, by noting that $\left|G L_{v}\left(\mathbb{F}_{p}\right)\right|=\prod_{j=1}^{v}\left(p^{j}-1\right) p^{\left(n^{2}-n\right) / 2}$, by a homogeneous space argument, letting $G L_{v}\left(\mathbb{F}_{p}\right)$ act on the subspaces, we have

$$
n_{v, l}=\frac{\left|G L_{v}\left(\mathbb{F}_{p}\right)\right|}{\left|G L_{l}\left(\mathbb{F}_{p}\right)\right|\left|G L_{v-l}\left(\mathbb{F}_{p}\right)\right| p^{l(v-l)}}=\left(\prod_{j=l+1}^{v}\left(p^{j}-1\right)\right) /\left(\prod_{j=1}^{v-l}\left(p^{j}-1\right)\right) .
$$

Example 4.14 For later work we will need the values of $\left|\Omega^{r}\left(\mathbb{F}_{p}^{v}\right)\right|$ in Table 4.

\begin{tabular}{|c|c|c|}
\hline$v$ & $r$ & $\left|\Omega^{r}\left(\mathbb{F}_{p}^{v}\right)\right|$ \\
\hline 1 & 2 & $p-1$ \\
\hline 1 & 3 & $(p-1)(p-2)$ \\
\hline 1 & 4 & $(p-1)\left(p^{2}-3 p+3\right)$ \\
\hline 1 & 5 & $(p-1)\left(p^{3}-4 p^{2}+6 p-4\right)$ \\
\hline 2 & 3 & $p(p-1)\left(p^{2}-1\right)$ \\
\hline 2 & 4 & $p(p-1)\left(p^{2}-1\right)\left(p^{2}+p-3\right)$ \\
\hline 2 & 5 & $p(p-1)\left(p^{2}-1\right)\left(p^{4}+p^{3}-3 p^{2}-4 p+6\right)$ \\
\hline 3 & 4 & $p^{3}(p-1)\left(p^{2}-1\right)\left(p^{3}-1\right)$ \\
\hline
\end{tabular}

Table 4: Values of $\left|\Omega^{r}\left(\mathbb{F}_{p}^{v}\right)\right|$ for small values of $r$ and $v$

Note that, since $G L_{v}\left(\mathbb{F}_{p}\right)$ acts freely by left multiplication on $\Omega^{r}\left(\mathbb{F}_{p}^{v}\right)$ then $\left|\Omega^{r}\left(\mathbb{F}_{p}^{v}\right)\right|$ is always evenly divided by $\left|G L_{v}\left(\mathbb{F}_{p}\right)\right|$. The $\left|\Omega^{r}\left(\mathbb{F}_{p}^{v}\right)\right|$ have been written to show this fact (some can be further factored). 
Example 4.15 Assume that $q: H^{\prime} \rightarrow G L_{v}\left(\mathbb{F}_{p}\right)$ is trivial. Then $H$ orbits determine a partition of $r, r=r_{1}+r_{2}+\cdots r_{s}$ with $1 \leq r_{1} \leq r_{2} \leq \cdots \leq r_{s}$. Such a partition of $r$

determines $\left(\begin{array}{c}r \\ r_{1}, \ldots, r_{s}\end{array}\right)$ partitions of $\{1, \ldots r\}$ with the canonical one being

$$
\left\{\left\{1, \ldots, r_{1}\right\},\left\{r_{1}+1, \ldots, r_{1}+r_{2}\right\}, \ldots,\left\{r_{1}+\cdots r_{s-1}+1, \ldots, r\right\}\right\} .
$$

A typical element of the stratum is obtained by selecting distinct nonzero $X_{1}, \ldots X_{S}$ satisfying

$$
\begin{aligned}
& r_{1} X_{1}+\cdots+r_{s} X_{s}=0 \\
& \operatorname{rank}\left[\begin{array}{lll}
X_{1} & \cdots & X_{s}
\end{array}\right]=v
\end{aligned}
$$

and then taking in order $r_{1}$ of $X_{1}, r_{2}$ of $X_{2}, \ldots r_{s}$ of $X_{S}$. To count the points fixed by $H$ we need the following generalization:

Proposition 4.16 Suppose that $a_{1}, \ldots, a_{s}$ are nonzero scalars in $\mathbb{F}_{p}$. Then the cardinality of the set

$$
\left\{\begin{array}{c|c}
X=\left[X_{1}, \ldots, X_{s}\right] \in \mathbb{F}_{p}^{v s} & \begin{array}{c}
X_{i} \neq 0, i=1, \ldots, s \\
a_{1} X_{1}+\cdots+a_{s} X_{s}=0, \operatorname{rank}(X)=v
\end{array}
\end{array}\right\}
$$

is $\left|\Omega^{v, s}\right|$.

Proof The map $\left[\begin{array}{lll}X_{1} & \cdots & X_{S}\end{array}\right] \longleftrightarrow\left[\begin{array}{lll}a_{1} X_{1} & \cdots & a_{S} X_{S}\end{array}\right]$ is a bijection between the given set and $\Omega^{v, r}$.

\subsection{Singular primes and Galois fusion}

The forgoing representation theory depends on certain divisibility properties of primes. The first problem occurs when a prime $p$ divides the order of $H^{\prime}$. Let us call such a prime a singular prime. The singular primes must satisfy $p \leq r$. The main problem is that complete reducibility of the representations $q$ and $\rho$ fails. Moreover there is new twist in that the sum over an orbit may be zero if the size of the orbit is divisible by the prime. In this case one needs to directly compute the representations and then compute the normalizers and fixed point set by brute force.

Another problem that can occur is that $\mathbb{F}_{p}$ may not be a splitting field for the representations $H^{\prime}$. In trying to describe the representations completely in terms of characters we will have to take characters together in Galois equivalent groupings in order that the representations $q$ be defined over the base field $\mathbb{F}_{p}$. We will call this Galois fusion. Furthermore, the formulas for the number of fixed points will have different polynomials in the primes. To help in describing this theory we use the following notation. For 
a prime $p$ and an integer $n$ let $U_{n}\left(\mathbb{F}_{p}\right)$ be the set of primitive $n^{\prime}$ th roots of 1 in $\mathbb{F}_{p}$ and let $\beta_{n}(p)=\left|U_{n}\left(\mathbb{F}_{p}\right)\right|$. Again, instead of developing the full theory here we will illustrate the ideas with the sample calculations in the next subsection.

\subsection{Rank 2 actions with 4 branch points}

Our general approach to compute the number of orbits with given $p$-rank and number of branch points takes the following steps.

(1) Determine all subgroups fixing at least one point, one representative for each conjugacy class. List them as $H_{1}, H_{2}, \ldots, H_{S}$ as described in Section 4.2. The sequence of groups will depend upon the prime.

(2) Compute $\left|\Omega^{H}\right|$ and $\left|N_{G}(H)\right|$ for each subgroup.

(3) Compute the matrix $D=\left[d_{i, j}\right]$ for the sequence of groups.

(4) Compute $\left|\left\langle\stackrel{\Omega}{\Omega}^{H}\right\rangle / G\right|$ for each subgroup using formula (27) and add up the results.

We will illustrate the steps by giving complete details for $p$-rank 2 and 4 branch points. For Step 1 we use a computer algebra system such as Magma [12] or GAP [14] to do the following:

- Determine a representative $H^{\prime}$ of each conjugacy class of $\Sigma_{4}$.

- Determine the character table of $H^{\prime}$.

- Decompose the reduced natural representation of $H^{\prime}$ into irreducibles over $\mathbb{C}$.

- Determine all compatible representations of $H^{\prime}$ and how they reduce over $\mathbb{F}_{p}$.

Remark 4.17 For each modular representation of an arbitrary group $G$ defined over $\mathbb{F}_{p}$ there is complex valued Brauer character defined on the $p$-regular elements of $G$ (order coprime to $p$ ). In the case $p \nmid|G|$ each Brauer character is an ordinary character and the modular representations of $G$ can be completely described by the irreducible characters. Thus in the discussion below we describe the modular characters by sums of irreducible ordinary characters from the character table of $G$.

Step 1 There are 11 conjugacy classes of subgroups of $\Sigma_{4}$. Using Magma [12], the orbits, and the decomposition of the reduced permutation (in terms of the characters of the subgroup), and the degrees of the representation are computed. The column in Table 5 giving the reduced permutation representation is written in terms of the characters of the subgroup using the order in the character table produced by Magma [12]. The list of the degrees is in the order given by the character table. The following notation is used: $\Sigma_{k}, A_{k}$ denote respectively the symmetric and alternating groups 


\begin{tabular}{|c|c|c|c|c|}
\hline$H^{\prime}$ & $\left|H^{\prime}\right|$ & orbits & red. perm. rep. & degrees \\
\hline$\Sigma_{1}^{4}$ & 1 & $\{1\},\{2\},\{3\},\{4\}$ & $3 \chi_{1}$ & 1 \\
\hline$\Sigma_{2} \times \Sigma_{1}^{2}$ & 2 & $\{1,2\},\{3\},\{4\}$ & $2 \chi_{1}+\chi_{2}$ & 1,1 \\
\hline$C_{2,2}$ & 2 & $\{1,2\},\{3,4\}$ & $\chi_{1}+2 \chi_{2}$ & 1,1 \\
\hline$A_{3} \times \Sigma_{1}$ & 3 & $\{1,2,3\},\{4\}$ & $\chi_{1}+\chi_{2}+\chi_{3}$ & $1,1,1$ \\
\hline$\Sigma_{2} \times \Sigma_{2}$ & 4 & $\{1,2\},\{3,4\}$ & $\chi_{1}+\chi_{3}+\chi_{4}$ & $1,1,1,1$ \\
\hline$V_{4}$ & 4 & $\{1,2,3,4\}$ & $\chi_{2}+\chi_{3}+\chi_{4}$ & $1,1,1,1$ \\
\hline$C_{4}$ & 4 & $\{1,2,3,4\}$ & $\chi_{2}+\chi_{3}+\chi_{4}$ & $1,1,1,1$ \\
\hline$\Sigma_{3} \times \Sigma_{1}$ & 6 & $\{1,2,3\},\{4\}$ & $\chi_{1}+\chi_{3}$ & $1,1,2$ \\
\hline$D_{4}$ & 8 & $\{1,2,3,4\}$ & $\chi_{3}+\chi_{5}$ & $1,1,1,1,2$ \\
\hline$A_{4}$ & 12 & $\{1,2,3,4\}$ & $\chi_{4}$ & $1,1,1,3$ \\
\hline$\Sigma_{4}$ & 24 & $\{1,2,3,4\}$ & $\chi_{4}$ & $1,1,2,3,3$ \\
\hline
\end{tabular}

Table 5: Subgroups of $\Sigma_{4}$

on $k$ points, $C_{k_{1}, \ldots, k_{s}}$ the cyclic group with cycle structure $\left(k_{1}, \ldots, k_{s}\right), D_{k}$ dihedral group on $k$ points and $V_{4}$ denotes the Klein 4 group.

Next we determine representatives of conjugacy classes of subgroups fixing a point, given in Table 6. Using Proposition 4.10, a stabilizer can be specified by a compatible character if one exists. Note that here we need to take away a one-dimensional character from the reduced representation to form $q$. This automatically eliminates $\Sigma_{4}, A_{4}$ and $\Sigma_{3} \times \Sigma_{1}$. If there is a fixed point of $H^{\prime}$ then $q$ must contain the trivial representation $\chi_{1}$. This eliminates $\Sigma_{3} \times \Sigma_{1}$ and taking $\chi_{1}$ away from the reduced representation of $A_{3} \times \Sigma_{1}$. Also in the $A_{3} \times \Sigma_{1}$ case, since we can take only one $\chi_{2}$ or $\chi_{3}$ then $\mathbb{F}_{p}$ must contain primitive cube roots of 1 . Different characters may lead to conjugate stabilizers. By the discussion in Section 4.4 if $\alpha$ normalizes $H^{\prime}$, and $g \in G L\left(2, \mathbb{F}_{p}\right)$ then the subgroup $H^{\prime}$ and $g q^{\alpha} g^{-1}$ determines a conjugate subgroup. The $N_{H^{\prime}} \chi$-orbits column in Table 6 lists the nontrivial orbits of characters under conjugation. If two linear combinations of characters are equivalent under the normalizer then conjugate stabilizers are determined and so we need only write one down. Furthermore, depending on the number of roots of unity in $\mathbb{F}_{p}$ some representations may not reduce completely. In that case the characters have to be taken together as a group, namely the set of all Galois conjugate characters. The nontrivial Galois orbits are noted in the Galois fusion column. Cases 11 and 11a are the same subgroup but are distinguished because $\left|\Omega^{H_{i}}\right|$ (see Table 7) depends on the value of $\beta_{4}(p)$. 


\begin{tabular}{|c|c|c|c|c|c|}
\hline Case & $H^{\prime}$ & $q$ & $N_{H^{\prime}} \chi$-orbits & Galois Fusion & restrictions \\
\hline 1 & $\Sigma_{1}^{4}$ & $2 \chi_{1}$ & & & \\
\hline 2 & $\Sigma_{2} \times \Sigma_{1}^{2}$ & $2 \chi_{1}$ & & & \\
\hline 3 & $\Sigma_{2} \times \Sigma_{1}^{2}$ & $\chi_{1}+\chi_{2}$ & & & \\
\hline 4 & $C_{2,2}$ & $\chi_{1}+\chi_{2}$ & & & \\
\hline 5 & $C_{2,2}$ & $2 \chi_{2}$ & & & $\beta_{3}(p)=2$ \\
\hline 6 & $A_{3} \times \Sigma_{1}$ & $\chi_{1}+\chi_{2}$ & $\left\{\chi_{2}, \chi_{3}\right\}$ & & \\
\hline 7 & $\Sigma_{2} \times \Sigma_{2}$ & $\chi_{1}+\chi_{3}$ & $\left\{\chi_{3}, \chi_{4}\right\}$ & & \\
\hline 8 & $\Sigma_{2} \times \Sigma_{2}$ & $\chi_{3}+\chi_{4}$ & $\left\{\chi_{3}, \chi_{4}\right\}$ & & \\
\hline 9 & $K_{4}$ & $\chi_{2}+\chi_{3}$ & $\left\{\chi_{2}, \chi_{3}, \chi_{4}\right\}$ & & $\beta_{4}(p)=2$ \\
\hline 10 & $C_{4}$ & $\chi_{2}+\chi_{3}$ & $\left\{\chi_{2}, \chi_{4}\right\}$ & & $\beta_{4}(p)=0$ \\
\hline 11 & $C_{4}$ & $\chi_{2}+\chi_{4}$ & $\left\{\chi_{2}, \chi_{4}\right\}$ & $\left\{\chi_{2}, \chi_{4}\right\}$ & $\beta_{4}(p)=2$ \\
\hline $11 a$ & $C_{4}$ & $\chi_{2}+\chi_{4}$ & $\left\{\chi_{2}, \chi_{4}\right\}$ & & \\
\hline 13 & $D_{4}$ & $\chi_{5}$ & & & \\
\hline
\end{tabular}

Table 6: Subgroups fixing a point, $p \neq 2,3$

Step 2 Next we list $\left|N_{G}\left(H_{i}\right)\right|$ and the $\left|\Omega^{H_{i}}\right|$. Each calculation of $\left|\Omega^{H_{i}}\right|$ was confirmed by using the brute force method using Maple [29]. The results are given in Table 7.

\begin{tabular}{|c|c|c|c|}
\hline Case & $\left|H^{\prime}\right|$ & $N_{G}\left(H_{i}\right)$ & $\left|\Omega^{H_{i}}\right|$ \\
\hline 1 & 1 & $24 p(p-1)\left(p^{2}-1\right)$ & $p(p-1)\left(p^{2}-1\right)\left(p^{2}+p-3\right)$ \\
\hline 2 & 2 & $4 p(p-1)\left(p^{2}-1\right)$ & $p(p-1)\left(p^{2}-1\right)$ \\
\hline 3 & 2 & $4(p-1)^{2}$ & $(p-1)\left(p^{2}-1\right)$ \\
\hline 4 & 2 & $8(p-1)^{2}$ & $(p-1)\left(p^{2}-1\right)$ \\
\hline 5 & 2 & $8 p(p-1)\left(p^{2}-1\right)$ & $p(p-1)\left(p^{2}-1\right)$ \\
\hline 6 & 3 & $3(p-1)^{2}$ & $(p-1)^{2}$ \\
\hline 7 & 4 & $4(p-1)^{2}$ & $(p-1)^{2}$ \\
\hline 8 & 4 & $8(p-1)^{2}$ & $(p-1)^{2}$ \\
\hline 9 & 4 & $8(p-1)^{2}$ & $(p-1)^{2}$ \\
\hline 10 & 4 & $4(p-1)^{2}$ & $p^{2}-1$ \\
\hline 11 & 4 & $8(p-1)^{2}$ & $(p-1)^{2}$ \\
\hline $11 a$ & 4 & $8\left(p^{2}-1\right)$ & $p-1$ \\
\hline 12 & 10 & $8(p-1)$ & \\
\hline
\end{tabular}

Table 7: Fixed point data for $\Sigma_{4}, p \neq 2,3$ 
Step 3 To compute the $d_{i, j}$ 's, let us first look at the case where all roots of unity are available $\beta_{3}(p)=\beta_{4}(p)=2$. The other cases may be derived from this case. In Table 8 , we present the $d_{i, j}$ 's, in matrix form with this ordering of the groups.

\begin{tabular}{|c|c|c|c|c|c|c|}
\hline$i$ & 1 & 2 & 3 & 4 & 5 & 6 \\
\hline Case & 1 & 2 & 3 & 4 & 5 & 6 \\
\hline$H_{i}^{\prime}$ & $\Sigma_{1}^{4}$ & $\Sigma_{2} \times \Sigma_{1}^{2}$ & $\Sigma_{2} \times \Sigma_{1}^{2}$ & $C_{2,2}$ & $C_{2,2}$ & $A_{3} \times \Sigma_{1}$ \\
\hline$q_{i}$ & $2 \chi_{1}$ & $2 \chi_{1}$ & $\chi_{1}+\chi_{2}$ & $\chi_{1}+\chi_{2}$ & $2 \chi_{2}$ & $\chi_{1}+\chi_{2}$ \\
\hline
\end{tabular}

\begin{tabular}{|c|c|c|c|c|c|c|}
\hline$i$ & 7 & 8 & 9 & 10 & 11 & 12 \\
\hline Case & 7 & 8 & 9 & 10 & $11 a$ & 12 \\
\hline$H_{i}^{\prime}$ & $\Sigma_{2} \times \Sigma_{2}$ & $\Sigma_{2} \times \Sigma_{2}$ & $K_{4}$ & $C_{4}$ & $C_{4}$ & $D_{4}$ \\
\hline$q_{i}$ & $\chi_{1}+\chi_{3}$ & $\chi_{3}+\chi_{4}$ & $\chi_{2}+\chi_{3}$ & $\chi_{2}+\chi_{3}$ & $\chi_{3}+\chi_{4}$ & $\chi_{5}$ \\
\hline
\end{tabular}

Table 8: Subgroup ordering

The values of $d_{i, j}=\left|D\left(H_{i}, H_{j}\right)\right|$ can be computed as follows. Using Magma [12] or GAP [14], find all the subgroup classes of $H_{j}^{\prime}$. For each subgroup class $\langle K\rangle, K \subseteq H_{j}^{\prime}$ find the corresponding $H_{i}$ 's such that $H_{i}^{\prime}$ is conjugate to $K$. Transport the character $q_{j}$ of $H_{j}$ to $H_{i}^{\prime}$ to determine the subgroup $H_{i}$. The entry for $d_{i, j}$ is then $|\langle K\rangle|$ (computed in $H_{j}^{\prime}$ ).

Example 4.18 Here is the matrix $D$ for the set of subgroups given in Table 8:

$$
\left[\begin{array}{ccccccccccccc} 
& H_{1} & H_{2} & H_{3} & H_{4} & H_{5} & H_{6} & H_{7} & H_{8} & H_{9} & H_{10} & H_{11} & H_{12} \\
H_{1} & 1 & 1 & 1 & 1 & 1 & 1 & 1 & 1 & 1 & 1 & 1 & 1 \\
H_{2} & & 1 & 0 & 0 & 0 & 0 & 1 & 0 & 0 & 0 & 0 & 0 \\
H_{3} & & 1 & 0 & 0 & 0 & 1 & 2 & 0 & 0 & 0 & 2 \\
H_{4} & & & 1 & 0 & 0 & 1 & 0 & 2 & 1 & 0 & 2 \\
H_{5} & & & & 1 & 0 & 0 & 1 & 1 & 0 & 1 & 1 \\
H_{6} & & & & & 1 & 0 & 0 & 0 & 0 & 0 & 0 \\
H_{7} & & & & & & 1 & 0 & 0 & 0 & 0 & 0 \\
H_{8} & & & & & & & & 1 & 0 & 0 & 0 & 1 \\
H_{9} & & & & & & & & 1 & 0 & 0 & 1 \\
H_{10} & & & & & & & & & 1 & 0 & 0 \\
H_{11} & & & & & & & & & & 1 & 1 \\
H_{12} & & & & & & & & & & & 1
\end{array}\right]
$$

Step 4 Finally we compute the number of orbits according to formula (27). We have $O^{\circ}=T D^{-1} N^{-1} L$ where $T$ and $N$ are the diagonal matrices formed from columns 
2 and 3 of Table 7, respectively, and $L$ is the vector formed from column 4 of Table 7. Accordingly we obtain the vector of orbits and the total number of orbits:

$$
\begin{aligned}
O^{\circ} & =\left[\begin{array}{llllllllllll}
\frac{p^{2}-8 p+7}{24} & 0 & \frac{p-3}{2} & \frac{p-5}{4} & 0 & 1 & 1 & 0 & 0 & 1 & 0 & 1
\end{array}\right]^{\top} \\
|\Omega / G| & =\frac{p^{2}+10 p+37}{24}
\end{aligned}
$$

We may check that all the numbers are integers by substituting in $p=12 k+1$, since 12 divides $p-1$.

There are a total of 4 families of primes which depend upon whether $\beta_{4}(p)=0,2$ and $\beta_{3}(p)=0,2$. For $p>3$ these families depend on the congruence class of $p$ mod 12. For each family certain subgroup cases from Table 5 are excluded. The corresponding matrices $D$ are obtained from the sample matrix above by deleting the row(s) and column(s) corresponding to the excluded subgroups. Depending on whether $\beta_{4}(p)=0,2$ one chooses the same subgroup $H_{10}$ but with a different normalizer and fixed point data given by cases 11 and 11a. The excluded subgroups are given in Table 9.

\begin{tabular}{|c|c|c|c|c|}
\hline$\beta_{3}(p)$ & $\beta_{4}(p)$ & $p$ mod 12 & excluded subgroups & $|\Omega / G|$ \\
\hline 0 & 0 & 11 & $H_{6}, H_{10}$ & $\frac{1}{24}\left(p^{2}+6 p+9\right)$ \\
\hline 2 & 0 & 7 & $H_{10}$ & $\frac{1}{24}\left(p^{2}+6 p+25\right)$ \\
\hline 0 & 2 & 5 & $H_{6}$ & $\frac{1}{24}\left(p^{2}+6 p+31\right)$ \\
\hline 2 & 2 & 1 & none & $\frac{1}{24}\left(p^{2}+6 p+37\right)$ \\
\hline
\end{tabular}

Table 9: Rank 2 actions with 4 branch points

The complete results for 3 and 4 branch points are given in Table 10 .

\section{Examples}

To finish, we illustrate our results through a number of interesting examples. To emphasize the explicit results which can be obtained, the first example we consider is very specific. Following this, we shall present some general examples which hold independent of the prime $p$.

Example 5.1 Let $G$ be an elementary abelian group of order 25. Using the RiemannHurwitz formula, it can be shown that there are exactly two different signatures for $\Gamma$ for a surface of genus 26 which give rise to subgroups of $\mathcal{M}_{26}$ isomorphic to $G-$ 
the signatures $(2 ;-)$ and $(1 ; 5,5,5)$. To find the total number of conjugacy classes of subgroups of $\mathcal{M}_{26}$ isomorphic to $G$, we need to find the number of classes induced by each of these signatures.

For the signature $(2 ;-)$, a direct application of Corollary 3.2 gives two different classes of subgroups. For the signature $(1 ; 5,5,5)$, there are two possibilities for the group $G^{h}$, either trivial or cyclic of order 5. If $G^{h}$ is trivial, $G^{e}=G$, so Proposition 4.1 implies there is just one epimorphism from $\Gamma$ onto $G$. Else, the image is cyclic of order 5 . In this case, we apply Corollary 2.9. For the hyperbolic part, observe that Corollary 3.2 tells us there will be a single epimorphism arising from $\Gamma$ with signature $(1 ;-)$ onto $G^{h}$. For the elliptic part, Example 5.2 below shows that all epimorphisms from $\Gamma$ with signature $(0 ; 5,5,5)$ onto $G^{e}$ are equivalent. Thus there is just one epimorphism arising from the elliptic part. Therefore, in total, there are $2+1+1 \times 1=4$ conjugacy classes of subgroups isomorphic to $G$ in $\mathcal{M}_{26}$.

Example 5.2 We can use our results to enumerate the equivalence classes of totally ramified actions for 3 or 4 branch points. Complete results for these cases are given in the Table 10.

\begin{tabular}{|c|c|c|c|c|}
\hline$r$ & $v$ & primes & congruence & \#equiv. classes \\
\hline 3 & 1 & 2 & & 0 \\
\hline 3 & 1 & 3 & & 1 \\
\hline 3 & 1 & $\beta_{3}(p)=0$ & $p=5 \bmod 6$ & $\frac{1}{6}(p+1)$ \\
\hline 3 & 1 & $\beta_{3}(p)=2$ & $p=1 \bmod 6$ & $\frac{1}{6}(p+5)$ \\
\hline 3 & 2 & all & & 1 \\
\hline 4 & 1 & 2,3 & & 1 \\
\hline 4 & 1 & $\beta_{4}(p)=0$ & $p=7,11 \bmod 12$ & $\frac{1}{24}\left(p^{2}+6 p+5\right)$ \\
\hline 4 & 1 & $\beta_{4}(p)=2$ & $p=1,5 \bmod 12$ & $\frac{1}{24}\left(p^{2}+6 p+17\right)$ \\
\hline 4 & 2 & 3 & & 2 \\
\hline 4 & 2 & $\beta_{3}(p)=0, \beta_{4}(p)=0$ & $p=11 \bmod 12$ & $\frac{1}{24}\left(p^{2}+10 p+9\right)$ \\
\hline 4 & 2 & $\beta_{3}(p)=2, \beta_{4}(p)=0$ & $p=7 \bmod 12$ & $\frac{1}{24}\left(p^{2}+10 p+25\right)$ \\
\hline 4 & 2 & $\beta_{3}(p)=0, \beta_{4}(p)=2$ & $p=5 \bmod 12$ & $\frac{1}{24}\left(p^{2}+10 p+21\right)$ \\
\hline 4 & 2 & $\beta_{3}(p)=2, \beta_{4}(p)=2$ & $p=1 \bmod 12$ & $\frac{1}{24}\left(p^{2}+10 p+37\right)$ \\
\hline 4 & 3 & all & & 1 \\
\hline
\end{tabular}

Table 10: Calculations for 3 and 4 branch points. Here $r$ is the number of branch points and $v$ is the $p$-rank 
The techniques and results developed in the previous examples allow us to develop some further results for general families of signatures and groups.

Example 5.3 Suppose $\Gamma$ has signature $(\rho ; p, p, p)$ with $p>3$ for any $\rho>0$ (see below for $\rho=0$ ), and let $G$ denote an elementary abelian group of order $p^{2}$. In this case, $G^{h}$ is either trivial or has order $p$. We first consider the case when $G^{h}$ has order $p$. By Example 5.2, there will be $\left(p+1+2 \beta_{3}(p)\right) / 6$ totally ramified epimorphisms onto $C_{p}$ and Corollary 3.2 implies there is a unique hyperbolic epimorphism onto $C_{p}$ giving a total of $1 \times\left(p+1+2 \beta_{3}(p)\right) / 6$. If $G^{h}$ is trivial, then Proposition 4.1 implies there exists a unique elliptic epimorphism. Thus there is a total of

$$
\frac{p+7+2 \beta_{3}(p)}{6}
$$

conjugacy classes of groups induced by this signature.

The results we have obtained for elementary abelian subgroups can in certain special cases be extended to provide information about other groups. We illustrate with the following two examples.

Example 5.4 By Proposition 4.1, there is a unique conjugacy class of elementary abelian subgroups of order $p^{2}$ in $\mathcal{M}_{(p-1)(p-2) / 2}$ with signature $(0 ; p, p, p)$. In fact, this can be derived explicitly by showing that any two epimorphisms $\eta_{1}, \eta_{2}: \Gamma \rightarrow G$ from $\Gamma$ with signature $(0 ; p, p, p)$ differ by an automorphism $\alpha \in \operatorname{Aut}(G), \eta_{1}=$ $\alpha \circ \eta_{2}$. The kernel $\operatorname{Ker}\left(\eta_{1}\right)$ is in fact a uniformizing surface group for the $p$ th Fermat curve with defining equation $x^{p}+y^{p}=1$ (see for example Wootton [34]). The full automorphism group of the $p$ th Fermat curve is isomorphic to the semidirect product $S_{3} \ltimes\left(C_{p} \times C_{p}\right) \cong \Gamma_{1} / \operatorname{Ker}\left(\eta_{1}\right)$ where $\Gamma_{1}$ has signature $(0 ; 2,3,2 p)$. By the uniqueness of $\operatorname{Ker}\left(\eta_{1}\right)$ and the uniqueness of the elementary abelian subgroup of $S_{3} \ltimes\left(C_{p} \times C_{p}\right)$, it follows that any two epimorphisms $\eta_{1}, \eta_{2}: \Gamma_{1} \rightarrow S_{3} \ltimes\left(C_{p} \times C_{p}\right)$ must differ by an automorphism $\alpha \in \operatorname{Aut}\left(S_{3} \ltimes\left(C_{p} \times C_{p}\right)\right)$. In particular, there will be a unique conjugacy class of subgroups of $\mathcal{M}_{(p-1)(p-2) / 2}$ isomorphic to $S_{3} \ltimes\left(C_{p} \times C_{p}\right)$ induced by $\Gamma$ with signature $(0 ; 2,3,2 p)$.

Example 5.5 Let $G$ be an elementary abelian group of order $p^{2}$ where $p \geq 5$ and suppose that $\Gamma$ has signature $(0 ; p, p, p, p)$. In Wootton [34] it is shown that there exists a unique epimorphism $\eta_{1}$ from $\Gamma_{1}$ with signature $(0 ; 2,2 p, 4)$ onto $H=D_{4} \ltimes\left(C_{p} \times C_{p}\right)$ (up to the action of Aut $(H)$ ) which restricts to an epimorphism $\left.\eta_{1}\right|_{\Gamma}: \Gamma \rightarrow C_{p} \times C_{p}$. In particular, this implies there exists a unique class of subgroups of $\mathcal{M}_{(p-1)^{2}}$ isomorphic to $H$ with signature $(0 ; 2,2 p, 4)$. Alternatively, this can also be seen on the level of generating vectors of the restriction $\left.\eta_{1}\right|_{\Gamma}$. Specifically, it can be shown that if $x$ and $y$ 
generate $G$, then all generating vectors for epimorphisms which extend to epimorphisms from $\Gamma_{1}$ to $H$ are Aut $(G) \times$ Aut $(\Gamma)$ equivalent to $\left(x, x^{-1}, y, y^{-1}\right)$. This implies there exists a unique class of groups isomorphic to $G$ in $\mathcal{M}_{(p-1)^{2}}$ contained in a class of subgroups isomorphic to $H$. Then, by the uniqueness of $G \leq H$, it follows that there exists a unique class of groups isomorphic to $H$ with signature $(0 ; 2,2 p, 4)$ in $\mathcal{M}_{(p-1)^{2}}$.

Observe that the generating vector in Example 5.5 is highly symmetric and this symmetry is reflected by the fact that $G$ is contained in a larger subgroup of $\mathcal{M}_{(p-1)^{2}}$. It seems plausible that if a generating vector is highly symmetric, then the corresponding conjugacy class of subgroups of the MCG will be contained in a class of larger finite subgroups of the MCG. This could lead to a new way to determine and enumerate classes of subgroups of the MCG which are not abelian using the methods we have developed for abelian subgroups.

\section{References}

[1] I Averill, J Gregoire, Tilings of Low-Genus Surfaces by Quadrilaterals (2002) RHIT Mathematical Sciences Technical Report 02-13 Available at http:// www.tilings.org/pubs/TRaverillgregoire.pdf

[2] L Bers, An extremal problem for quasiconformal mappings and a theorem by Thurston, Acta Math. 141 (1978) 73-98 MR0477161

[3] J S Birman, Braids, links, and mapping class groups, Annals of Mathematics Studies 82, Princeton University Press, Princeton, N.J. (1974) MR0375281

[4] T E Brendle, The Torelli Group and Representations of Mapping Class Groups, $\mathrm{PhD}$ thesis, Columbia University (2002)

[5] T E Brendle, B Farb, Every mapping class group is generated by 6 involutions, J. Algebra 278 (2004) 187-198 MR2068073

[6] T Breuer, Characters and automorphism groups of compact Riemann surfaces, London Mathematical Society Lecture Note Series 280, Cambridge University Press, Cambridge (2000) MR1796706

[7] S A Broughton, The equisymmetric stratification of the moduli space and the Krull dimension of mapping class groups, Topology Appl. 37 (1990) 101-113 MR1080344

[8] S A Broughton, Classifying finite group actions on surfaces of low genus, J. Pure Appl. Algebra 69 (1991) 233-270 MR1090743

[9] S A Broughton, Normalizers and centralizers of elementary abelian subgroups of the mapping class group, from: "Topology '90 (Columbus, OH, 1990)", Ohio State Univ. Math. Res. Inst. Publ. 1, de Gruyter, Berlin (1992) 77-89 MR1184404 
[10] S A Broughton, Enumeration of the Equisymmetric Strata of the Moduli Space of Surfaces of Low Genus (2005) Preliminary report Available at http:// www.rose-hulman.edu/ brought/Epubs/SantaBarbara/santabarbara.html

[11] S A Broughton, R Dirks, M Sloughter, C Vinroot, Triangular Surface Tiling Groups for Genus 2 to 13 (2002) RHIT Mathematical Sciences Technical Report 01-01 Available at http://www.tilings.org/pubs/tileclass.pdf

[12] J Cannon, W Bosma (editors), Handbook of Magma Functions, Edition 2.13 (2006)

[13] MDE Conder, RS Kulkarni, Infinite families of automorphism groups of Riemann surfaces, from: "Discrete groups and geometry (Birmingham, 1991)", London Math. Soc. Lecture Note Ser. 173, Cambridge Univ. Press, Cambridge (1992) 47-56 MR1196915

[14] GAP Group, GAP - Groups, Algorithms and Programming, Version 4.4 (2006) Available at http://www.gap-system.org/

[15] J Gilman, Prime Order Automorphisms of Riemann Surfaces arXiv: math.GT/0701286v1

[16] J Gilman, On conjugacy classes in the Teichmüller modular group, Michigan Math. J. 23 (1976) 53-63 MR0430320

[17] J Gilman, A matrix representation for automorphisms of compact Riemann surfaces, Linear Algebra and Appl. 17 (1977) 139-147 MR0499135

[18] J Gilman, Structures of elliptic irreducible subgroups of the modular group, Proc. London Math. Soc. (3) 47 (1983) 27-42 MR698926

[19] J Gilman, D Patterson, Intersection matrices for bases adapted to automorphisms of a compact Riemann surface, from: "Riemann surfaces and related topics: Proceedings of the 1978 Stony Brook Conference (State Univ. New York, Stony Brook, N.Y., 1978)", Ann. of Math. Stud. 97, Princeton Univ. Press, Princeton, N.J. (1981) 149-166 MR624812

[20] W J Harvey, Cyclic groups of automorphisms of a compact Riemann surface, Quart. J. Math. Oxford Ser. (2) 17 (1966) 86-97 MR0201629

[21] W J Harvey, On branch loci in Teichmüller space, Trans. Amer. Math. Soc. 153 (1971) 387-399 MR0297994

[22] D Johnson, A survey of the Torelli group, from: "Low-dimensional topology (San Francisco, CA, 1981)", Contemp. Math. 20, Amer. Math. Soc., Providence, RI (1983) 165-179 MR718141

[23] S P Kerckhoff, The Nielsen realization problem, Ann. of Math. (2) 117 (1983) 235-265 MR690845

[24] R S Kulkarni, Infinite families of surface symmetries, Israel J. Math. 76 (1991) 337-343 MR1177349 
[25] A M Macbeath, The classification of non-euclidean plane crystallographic groups, Canad. J. Math. 19 (1967) 1192-1205 MR0220838

[26] A M Macbeath, Generators of the linear fractional groups, from: "Number Theory (Proc. Sympos. Pure Math., Vol. XII, Houston, Tex., 1967)”, Amer. Math. Soc., Providence, R.I. (1969) 14-32 MR0262379

[27] C Maclachlan, Abelian groups of automorphisms of compact Riemann surfaces, Proc. London Math. Soc. (3) 15 (1965) 699-712 MR0179348

[28] C Maclachlan, Modulus space is simply-connected, Proc. Amer. Math. Soc. 29 (1971) 85-86 MR0286995

[29] Maplesoft, Maple, Version 10 Available at http://www.maplesoft.com/

[30] D Singerman, Subgroups of Fuschian groups and finite permutation groups, Bull. London Math. Soc. 2 (1970) 319-323 MR0281805

[31] D Singerman, Finitely maximal Fuchsian groups, J. London Math. Soc. (2) 6 (1972) 29-38 MR0322165

[32] D Singerman, Symmetries of Riemann surfaces with large automorphism group, Math. Ann. 210 (1974) 17-32 MR0361059

[33] D Singerman, Automorphisms of maps, permutation groups and Riemann surfaces, Bull. London Math. Soc. 8 (1976) 65-68 MR0393209

[34] A Wootton, Non-normal Bely̌ p-gonal surfaces, from: "Computational aspects of algebraic curves”, (T Shaska, editor), Lecture Notes Ser. Comput. 13, World Sci. Publ., Hackensack, NJ (2005) 95-108 MR2182036

[35] A Wootton, The full automorphism group of a cyclic p-gonal surface, J. Algebra 312 (2007) 377-396 MR2320463

[36] H Zieschang, E Vogt, H-D Coldewey, Surfaces and planar discontinuous groups, Lecture Notes in Mathematics 835, Springer, Berlin (1980) MR606743 Translated from the German by John Stillwell

Department of Mathematics, Rose Hulman Institute of Technology

Terre Haute, IN 47803, USA

Department of Mathematics, The University of Portland

Portland, OR 97203, USA

allen.broughton@rose-hulman.edu, wootton@up.edu

http://www.rose-hulman.edu/ brought/, http://faculty.up.edu/wootton/

Received: 21 November 2006 Revised: 13 September 2007 